\title{
Política social y desigualdad en la región del Gran Buenos Aires, Argentina, 1992-2012
}

\author{
Agustín Salvia, Santiago Poy y Julieta Vera
}

\author{
Universidad de Buenos Aires, Argentina
}

\section{Resumen}

El presente trabajo analiza en qué medida las políticas sociales - tomando en consideración los programas de asistencia social directa, los planes de protección al desempleo y las transferencias previsionales (jubilaciones y pensiones) - afectaron la distribución de ingresos familiares en la región del Gran Buenos Aires (Argentina) durante las últimas dos décadas. Para desarrollar este análisis se aplicó un ejercicio de descomposición del coeficiente de desigualdad de Gini. Se utilizaron las bases de la Encuesta Permanente de Hogares (EPH) del Instituto Nacional de Estadísticas y Censos (INDEC) correspondientes a la Región del Gran Buenos Aires. La evidencia presentada da cuenta de los límites que enfrentan las políticas sociales redistributivas para resolver por sí solas las desigualdades estructurales que presenta el sistema productivo y el mercado de trabajo.

Palabras clave: Desigualdad, Región del Gran Buenos Aires, políticas de transferencias de ingreso, programas de empleo, descomposición del Coeficiente de Gini.

\section{Abstract}

Social policy and inequality in the Greater Buenos Aires, Argentina, 1992-2012

This research aims to examine how social policies — considering income transfers, employment programs and pension and retirement income- affected the income distribution in the Greater Buenos Aires (Argentina) during the last two decades. To that end, a decomposition analysis of the Gini coefficient is presented. Data come from a household survey known as Encuesta Permanente de Hogares (EPH), carried out by the National Institute for Statistics and Censuses (INDEC) focused on the Greater Buenos Aires (GBA). The evidence presented reveals the limits that redistributive social policies face to resolve by themselves, the structural inequalities from the productive system and the labor market.

Key words: Inequality, Greater Buenos Aires, cash transfers policies, employment programs, decomposition of the Gini Coefficient. 


\section{INTRODUCCIÓN}

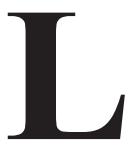

a superación de los problemas distributivos continúa siendo objeto de interés tanto en América Latina como en el resto de las regiones subdesarrolladas del mundo. Este artículo se interesa por el papel que tienen las "políticas sociales"1 en los procesos distributivos. Se trata de un tema relevante ya que durante las últimas décadas se produjo en varios países latinoamericanos una fuerte expansión del gasto social y una caída de la desigualdad en la distribución del ingreso (CEPAL, 2009; CEPAL, 2013; Lustig et al., 2011; Gasparini et al., 2011; Levinas, 2014; Medina y Galván, 2014).

Al respecto, resulta significativo registrar que coinciden en esta lectura tanto el enfoque estructuralista de la Comisión Económica para América Latina y el Caribe (CEPAL, 2014) como la perspectiva ortodoxa del Banco Mundial (Birdsall y de la Torre, 2008; Ferreira et al., 2013). La desigualdad habría caído sobre todo en la última década no sólo como resultado de la mayor demanda agregada de empleo, la reducción de la tasa de dependencia de los hogares y el aumento de las trasferencias de ingresos, sino también - y de manera principal — por una reducción del diferencial salarial entre trabajadores calificados y no calificados (Lustig et al., 2011; CEPAL, 2014)

Asimismo, ambos enfoques parecen también coincidir en la actual etapa post-reformas en el nuevo esquema de desarrollo que deben adoptar las economías de la región: la reducción de la pobreza y la desigualdad constituyen condiciones necesarias del crecimiento (Birdsall y de la Torre, 2001; De Ferranti et al., 2003; Perry et al., 2006; CEPAL, 2013). Sin embargo, no menos importante es reconocer que estos aparentes acuerdos esconden diferencias teórico-políticas de fondo. ${ }^{2}$

Es suficiente comprender hasta aquí que para la ortodoxia del Banco Mundial, el crecimiento con reducción de la pobreza se ha logrado en el contexto de una economía mundial inestable pero suficientemente globalizada, donde las ventajas comparativas de la región parecen estar dando frutos gracias a las reformas estructurales. En este marco, la sociedad se ha

\footnotetext{
${ }^{1}$ Se adopta aquí una forma ampliamente difundida pero no menos difusa de concebir a las "políticas sociales" como el conjunto de dispositivos a partir de los cuales el Estado interviene de manera directa en la redistribución secundaria del ingreso, en oposición a la distribución primaria que estaría dominada por el funcionamiento de los mercados (Isuani, 2008).

${ }_{2}^{2}$ Para una revisión de estas diferencias teóricas y político-ideológicas alrededor del vínculo entre relaciones de clase, relaciones de poder, crecimiento, desigualdad y pobreza, ver Cortés (2013, 2014).
} 
hecho más fluida y han crecido las clases medias, pero es hora de bajar aún más la pobreza y mejorar la productividad invirtiendo en capital humano, promoviendo la igualdad de oportunidades y aumentando la competencia social. Para ello es necesario profundizar y extender las reformas liberalizadoras al campo comercial, fiscal, educativo, laboral, crediticio, político y social. En este marco, los programas sociales condicionados de transferencia de ingresos orientados a los pobres tienen un papel crucial (Birdsall y de la Torre, 2001; De Ferranti et al., 2003; Perry et al., 2006; Ferreira et al., 2013).

Por su parte, para la heterodoxia de la CEPAL, la mejor performance de América Latina se ha asentado en los esfuerzos puestos por los gobiernos para orientar los excedentes del comercio exterior y la disponibilidad de capitales internacionales hacia la inversión en infraestructura, el crecimiento del mercado interno y la expansión del gasto social. La preocupación hacia adelante es que este modelo sigue siendo sumamente vulnerable frente a la trampa de crecimiento que brinda la especialización productiva y la concentración económica, ambas estrechamente relacionadas con el mercado internacional. Según la teoría estructuralista latinoamericana, un modelo de esta naturaleza se sostiene en la desigual penetración y distribución de los avances tecnológicos y recursos productivos, teniendo esto como consecuencia necesaria una mayor heterogeneidades estructurales a nivel ocupacional, social y territorial; frente a lo cual muy poco pueden hacen las políticas sociales redistributivas (Prebisch, 1949; Pinto, 1973; Rodríguez, 2006; CEPAL, 2014). ${ }^{3}$

Es decir, no es aconsejable perder de vista la evidencia de que el horizonte de la convergencia en un desarrollo con equidad no ha dejado de ser un proyecto todavía lejano. Cabe señalar que en la historia argentina reciente confluyen las reformas estructurales neoliberales de los años noventa (1992-2001), la crisis de dicho modelo en 2001-2002 y las políticas "neo-desarrollistas" de reactivación y pro-mercado interno de la última década (2003-2012).

En un contexto de cambio cualitativo entre la política social del período neoliberal y la etapa "neo-desarrollista" post-crisis, el presente trabajo pretende indagar sobre la relevancia que tienen los nuevos programas de transferencia de ingresos, en comparación con los sistemas tradicionales de la seguridad social y el propio mercado de trabajo, como instrumentos

\footnotetext{
${ }^{3} \mathrm{Al}$ respecto, Prebisch (1963) destaca que la penetración desigual de la tecnología sustrae a una considerable proporción de la población económicamente activa del crecimiento; y que el problema del desarrollo consiste esencialmente en crear las condiciones para mejorar su asimilación a un esquema más productivo y más integrado.
} 
para crear un mínimo de inclusión social, evaluando así sus efectos en el índice de desigualdad en la distribución del ingreso.

Para llevar adelante este análisis se utiliza información sobre los ingresos de los hogares según fuentes, a partir de micro datos de la Encuesta Permanente de Hogares (EPH) a cargo del Instituto Nacional de Estadísticas y Censos (INDEC). Por motivos de fiabilidad y comparabilidad, el análisis de micro datos se limitó al área metropolitana del Gran Buenos Aires (GBA) ${ }^{4}$ Los años seleccionados corresponden a las ondas del mes de mayo de 1992, 1998 y 2003 (correspondientes a la EPH "puntual") y el tercer trimestre de 2003, primer trimestre de 2004 y segundos trimestres de los años 2005 a 2012 (correspondientes a micro datos de la EPH "continua"). ${ }^{5}$ Los ejercicios realizados para la compatibilización de ambas fuentes de datos se detallan en un anexo al final del documento. ${ }^{6}$

Ahora bien, cabe aclarar que en los análisis aquí propuestos, la información empírica empleada remite - en primer lugar - a estáticas comparadas y — en segundo lugar - contiene un nivel de complejidad metodológica tal que el empalme de datos hubiese sido más distorsivo y, por ende, menos fiable. Por este motivo, el análisis se desarrolló a través de una serie "cortada" de resultados, evaluando de manera independiente los procesos ocurridos durante los distintos períodos político-económico considerados. Mientras que las primeras fases político-económicas se trabajan con datos provenientes de la EPH "puntual", el periodo de políticas "heterodoxas" es estudiado con datos obtenidos a partir de la EPH "continua". Se consideró que este procedimiento ofrece resultados de mayor validez y fiabilidad.

En la primera sección se presenta una descripción general de los cambios ocurridos en la orientación de las políticas sociales durante las últimas dos décadas en la Argentina. En la segunda sección se hace una cuantificación de los alcances e incidencia de las transferencias directas generadas por el gasto social (ingresos de la seguridad social y programas de lucha

\footnotetext{
${ }^{4} \mathrm{Si}$ bien los resultados de este trabajo no tienen alcance nacional, el área metropolitana del Gran Buenos Aires concentra casi $30 \%$ de la población urbana del país, consume aproximadamente 40 por ciento de la energía eléctrica demandada a nivel nacional y produce más 45 por ciento del PBG nacional. De tal manera que los eventos ocurridos en esta jurisdicción impactan considerablemente en cualquier análisis del país en su conjunto.

${ }^{5}$ Los años y ondas/trimestres seleccionados corresponden a aquellos considerados de mayor estabilidad, significatividad en términos de procesos históricos y confiables en cuanto a los resultados obtenidos. En la EPH "continua" se priorizó la utilización del segundo trimestre de cada año de modo tal de lograr mayor similitud con el criterio empleado en la EPH "puntual" (ondas de mayo). Las excepciones en este sentido corresponden al año 2003 (para la cual la EPH "continua" está disponible a partir del tercer trimestre) y al año 2004 (debido a limitaciones y baja confiabilidad de los microdatos del segundo trimestre en lo que respecta a las fuentes de ingresos acá consideradas).

${ }^{6}$ Para mayores detalles acerca de los cambios en la fuente de información y los ajustes metodológicos realizados para permitir la comparación interanual, véase también Salvia y Vera (2012).
} 
contra la pobreza) sobre el presupuesto de los hogares, así como su efecto neto "inmediato" sobre la desigualdad distributiva. El tercer apartado evalúa el impacto de dichas transferencias sobre los cambios en la desigualdad a través de la descomposición del coeficiente de Gini. Por último, en el cuarto apartado se discuten los resultados a la luz de las preocupaciones que motivaron este trabajo.

\section{TranSFORMACIÓN EN LA REDISTRIBUCIÓN SECUNDARIA DEL INGRESO DE LAS POLÍTICAS SOCIALES UNIVERSALES Y ASISTENCIALISTAS}

Los cambios ocurridos a partir de los años setenta en América Latina implicaron no sólo una redefinición del estilo de crecimiento sino también de las intervenciones sociales del Estado La centralización de los sistemas de política social cedió espacio a la descentralización; mientras que la universalización dio paso a una creciente focalización. Al respecto, un aspecto crucial es la integración social de los sectores excluidos, más que la integración sistémica de las relaciones sociales de producción que generaban tales sobrantes poblacionales. ${ }^{7}$

En este contexto, las políticas tradicionales de seguridad social experimentaron un cambio dirigido a generar la intervención de programas masivos de asistencia directa "transitoria" dirigida a poblaciones de riesgo. Surgió así, con el apoyo técnico-financiero de organismos multinacionales de crédito (Banco Mundial, BID), una serie de nuevos programas de transferencia de ingresos (Barrientos y Hulme, 2008; Barba y Cohen, 2011; CEPAL, 2009).

Estos programas no constituyen derechos permanentes sino que son definidos como "transitorios". Las poblaciones beneficiarias reciben transferencias mientras se cumplan condiciones de elegibilidad y/o permanencia en el programa; y hasta tanto la misma dinámica económica de los mercados no sea capaz de absorberlas. Estos programas se proponen dotar de activos - tanto en capital social como capital humano - a las poblaciones objeto de beneficio. Esto debido a que se busca una mayor integración de estos sectores a las relaciones de mercado, siendo que el diagnóstico es que estas poblaciones no participan del mercado de trabajo formal no porque exista segmentación o la demanda sea insuficiente sino porque su capital humano-institucional no se ajusta a la demanda (Perry et al., 2006).

\footnotetext{
${ }^{7}$ Esta contradicción fue planteada varias décadas atrás por Nun y el equipo de investigación a su cargo en 1969 cuando planteó que la "masa marginal" - en contaste con el ejército industrial de reserva clásico - era una manifestación del bajo grado de "integración sistémica" que generaba el desarrollo capitalista desigual y dependiente en su fase monopólica.
} 
En la Argentina puede apreciarse una evolución semejante, aunque fundada en condiciones de protección pre-reformas estructurales algo más extendidas que en otros países de la región. El país tenía hasta la década del setenta un sistema de política social basado en un régimen universal estratificado formado por tres componentes: la provisión universalista de los sistemas de educación y salud públicas; las prestaciones de salud y previsionales diferenciadas según nivel salarial y rama de actividad; y la atención localizada a los problemas de pobreza extrema y falta de vivienda (Lo Vuolo y Barbeito, 1998).

El incremento de la pobreza extrema y del desempleo durante los ochenta puso de manifiesto la incapacidad de los servicios de protección social del llamado "estado benefactor" para atender las nuevas demandas sociales resultantes del estancamiento económico y el deterioro del mercado de trabajo (Grassi et al., 1994; Lo Vuolo y Barbeito, 1998). Cada uno de los componentes mencionados comenzó a experimentar problemas de financiamiento y de gestión que impulsaron importantes iniciativas orientadas a la redefinición de la política social. ${ }^{8}$

En ese contexto, las reformas de inspiración neoliberal de los años noventa introdujeron cambios en las políticas sociales tendientes a reducir el déficit público generado por los gastos universales. Asimismo, la orientación neoliberal de las políticas adoptadas en los años noventa incentivó la participación del sector privado en el mercado de los servicios sociales universales, así como también buscó asistir de manera focalizada a los "grupos vulnerables" o "grupos de riesgo". Estas reformas incrementaron la importancia de las políticas de asistencia directa, aumentaron la inequidad en los servicios prestados en forma universal (a través de la descentralización de los servicios educativos y sanitarios a las jurisdicciones sub-nacionales) y profundizaron el carácter estratificado y fragmentado de aquellos servicios que ya presentaban esos rasgos (vía la desregulación de las obras sociales y la introducción del régimen de capitalización en el sistema de la seguridad social) (Grassi, 2003; Repetto y Andrenacci, 2005; Falappa y Andrenacci, 2008; Danani y Grassi, 2008).

Pero la crisis terminal en 2001-2002 del régimen de libre convertibilidad cambiaria —instaurado en 1992 - estuvo acompañada por el abrupto aumento del desempleo, la pobreza y la desigualdad. ${ }^{9}$ Se evidenciaron las

\footnotetext{
${ }^{8}$ A partir de los años setenta el modelo que atribuía al Estado un rol central en la redistribución de bienes sociales enfrentó desafíos político-económicos que pusieron en cuestión su supervivencia. La literatura especializada presenta disidencias respecto de las razones que habrían provocado su crisis (Grassi et al., 1994; Bonvecchi y Smulovitz, 2008; Isuani, 2008).

${ }^{9}$ Sobre la evolución de los indicadores sociales durante y a partir de la crisis, véase, entre otros, Lindenboim (2012).
} 
limitaciones del sistema de protección y asistencia social generado por las reformas estructurales. A partir de ese momento, bajo un modelo político opuesto al esquema neoliberal, tuvo lugar un vigoroso proceso de reactivación económica con fuerte demanda del empleo y promoción de actividades vinculadas al mercado interno (Damill y Frenkel, 2006; Damill et al., 2011; CIFRA, 2011).

Entre 2003 y 2012, se aplicaron "contra reformas" en materia de políticas laborales, políticas de ingresos y políticas de la seguridad social (Novick, 2006; Palomino, 2007; Bertranou y Paz, 2007; Danani y Grassi, 2008; Neffa y Panigo, 2009; Danani y Hintze, 2011). Estas medidas estuvieron acompañadas por un fuerte aumento de los gastos en infraestructura social y servicios públicos de educación y salud, así como también en materia de previsión social (jubilaciones y pensiones); manteniéndose al mismo tiempo vigente y/o ampliándose según la coyuntura, los programas de provisión y asistencia directa de ingresos.

En general, se destaca el cambio de rumbo de la política social - a partir de 2003-, fundamentando por lo general esta afirmación en los cambios normativos, el aumento registrado por el gasto social y la ampliación de los programas sociales de transferencia de ingresos. A partir de estas evidencias se afirma que la política social ha pasado a tener - a diferencia del período neoliberal - un papel activo en la transferencia de recursos económicos a favor de los sectores medios y más pobres; explicando ello la reducción ocurrida en las tasas de indigencia/pobreza, así como la mejora en la distribución del ingreso. Justamente, son estas afirmaciones las que serán objeto de análisis en los siguientes apartados.

\section{LOS PROGRAMAS DE PROTECCIÓN CONTRA LA POBREZA Y LA PREVISIÓN SOCIAL. IMPACTO SOBRE EL INGRESO FAMILIAR Y LA DESIGUALDAD}

En este apartado interesa evaluar el impacto específico que tuvieron las transferencias previsionales (el sistema de jubilaciones y pensiones) y los instrumentos de promoción y asistencia social directa - caracterizados como programas de protección contra la pobreza- sobre los niveles de ingreso familiar y la desigualdad bajo distintas fases político-económicas del periodo 1992-2012.

Resulta de interés dar cuenta de la incidencia y la evolución en materia de cobertura y participación en el presupuesto familiar de las dos fuentes mencionadas de transferencia de ingresos en materia de política social: i) el sistema previsional (jubilaciones y pensiones), y ii) los "programas de protección contra la pobreza" señalados en el apartado anterior (programas 
de protección al desempleo y programas de asistencia social directa). Esta evolución puede verse en la Figura 1 y en la Figura 2, en los cuales sobresalen los principales rasgos asociados a cada fase político-económica.

1. Entre los años 1992 y 1998, descendió levemente la proporción de hogares perceptores de ingresos del sistema de seguridad social — jubilaciones o pensiones - al mismo tiempo que aumentó la participación de este tipo de cobertura en el presupuesto familiar. ${ }^{10}$ Asimismo, se incrementó la proporción de unidades domésticas que recibían algún programa social así como la incidencia de este tipo de ingresos en el presupuesto de los hogares, estando estos procesos vinculados a cierta expansión de programas de protección al desempleo durante este periodo. ${ }^{11}$

2. Entre 1998 y 2003, en una fase recesiva y de crisis económico-social con posterior recuperación a partir del año 2002, los datos dan cuenta del abrupto incremento en el porcentaje de hogares perceptores de programas de protección al desempleo y, adicionalmente, de la participación de estos ingresos en el presupuesto familiar. La implementación, a partir del año 2002, del "Programa Jefas de Hogar Desocupados (PJHD)" estaría explicando estas tendencias. Adicionalmente, se observa que si bien desciende la cantidad de hogares con ingresos por jubilación o pensión, se incrementó — durante estos años - la participación de este tipo de ingresos en el presupuesto familiar de los hogares. ${ }^{12}$

3. En el período 2003-2007 —en un contexto de fuerte reactivación económica y mejora de los indicadores económicos, laborales y sociales-, tuvo lugar una activa política gubernamental en materia de ingresos, en la cual ganaron preponderancia las transferencias directas de ingresos (contributivas y no contributivas), a la vez que disminuyó la participación de los programas de protección contra el desempleo: bajó la proporción de hogares con planes de empleo y se incrementó el porcentaje de unidades domésticas que percibían algún tipo de progra-

${ }^{10}$ La mayor importancia relativa que adquieren las jubilaciones y pensiones en el presupuesto de los hogares está vinculada al crecimiento de la masa de ingresos en esta fuente; asociado al aumento de los ingresos medios superior al promedio de la economía.

${ }^{11}$ Al respecto, cabe recordar la puesta en marcha —en 1996- del Plan Trabajar y otros programas de protección contra el desempleo, en un contexto de reformas estructurales que promueven la flexibilización de los mecanismos regulatorios del mercado de trabajo y, a su vez, en el marco de una política social asistencial focalizada en los individuos con problemas de inserción en el mercado laboral.

${ }^{12}$ En un periodo fuertemente recesivo y de pérdidas generalizadas de ingresos, las jubilaciones y pensiones si bien no escapan a la evolución desfavorable del conjunto de la economía, evidencian un descenso de los ingresos medios de menor intensidad respecto a otras fuentes. 
ma de asistencia social directa. Estas tendencias fueron acompañadas por cambios —en el mismo sentido - en la conformación del presupuesto familiar. A su vez, el aumento de la jubilación mínima, así como la implementación de la moratoria y la jubilación anticipada permitió aumentar de manera significativa la tasa de cobertura del sistema previsional y la relevancia relativa de estos ingresos en el presupuesto de los hogares.

4. En el último período analizado (2007-2012) —en el cual tuvo lugar un proceso de desaceleración del crecimiento económico- continuaron fortaleciéndose las políticas activas de ingresos con eje en la recomposición salarial, en el sistema previsional y en la puesta en marcha de un programa que universaliza las transferencias monetarias por hijo al ámbito de los desocupados y trabajadores informales (Asignación Universal por Hijo). Esto se reflejó en el crecimiento de los hogares con ingresos por jubilaciones/pensiones o programas de asistencia directa, así como también en el aumento de la participación de estas fuentes de ingreso en el presupuesto familiar.

Resulta pertinente destacar la muy baja participación de los programas sociales de lucha contra la pobreza en el total de los ingresos familiares más allá del período político-económico y las diferentes características de los mismos durante la fase de reformas estructurales, la crisis de la convertibilidad o durante la recuperación posterior bajo políticas heterodoxas.

Ahora bien, ¿cuál fue el impacto directo o "instantáneo" de estas intervenciones públicas sobre el ingreso y su distribución? Para responder a este interrogante, la Figura 3 exhibe la media de ingresos per cápita (IPCF) - a valores constantes del año 2012 - considerando y excluyendo las fuentes de ingresos provenientes de programas de asistencia social directa y de protección al desempleo, y de aquellos provenientes del sistema de jubilaciones y pensiones. En igual sentido, la Figura 4 da cuenta de cuál hubiese sido el coeficiente de desigualdad de Gini si esas transferencias no hubiesen existido.

El supuesto que está detrás de este análisis es que si los sistemas de transferencia de ingreso tienen como meta una más equitativa redistribución secundaria del ingreso, en la medida que esto ocurra habrá hogares situados en los estratos más desfavorecidos de la estructura social que mejorarán su ingreso familiar. 


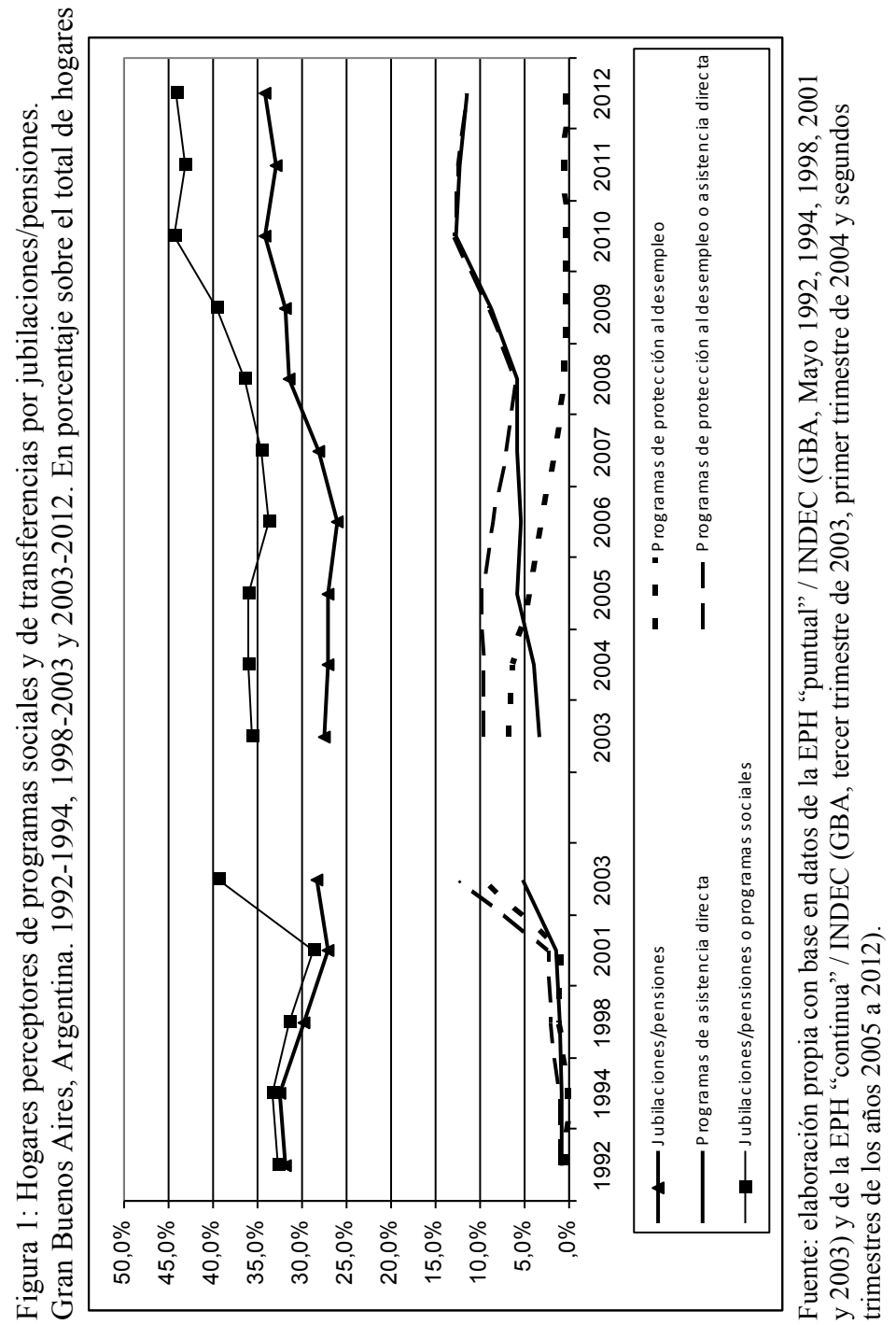


Política social y desigualdad en la región del Gran Buenos Aires, Argentina, 1992-2012/A. SALVIA, POY y J. VERA

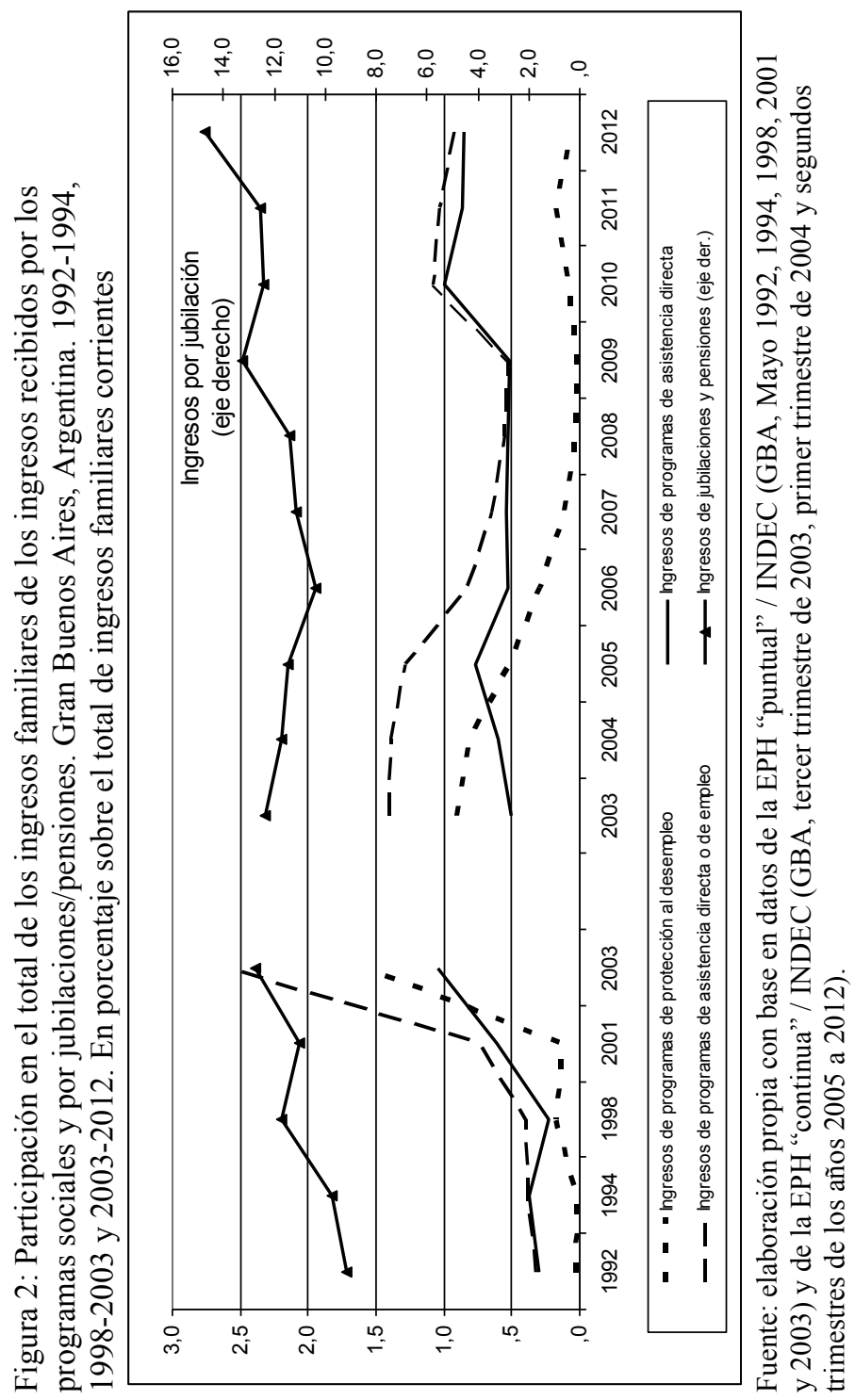




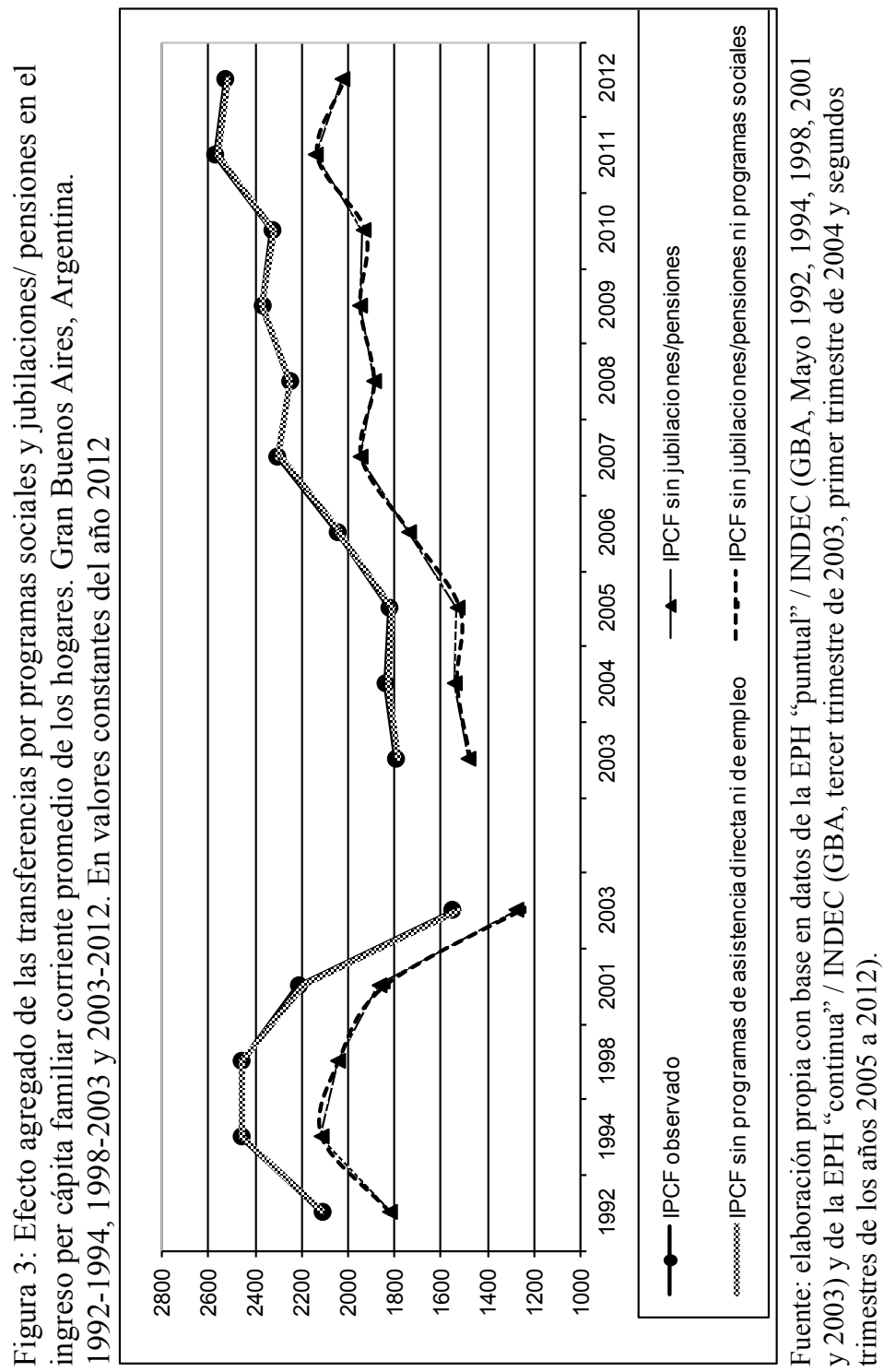


Política social y desigualdad en la región del Gran Buenos Aires, Argentina, 1992-2012/A. SALVIA, POY y J. VERA

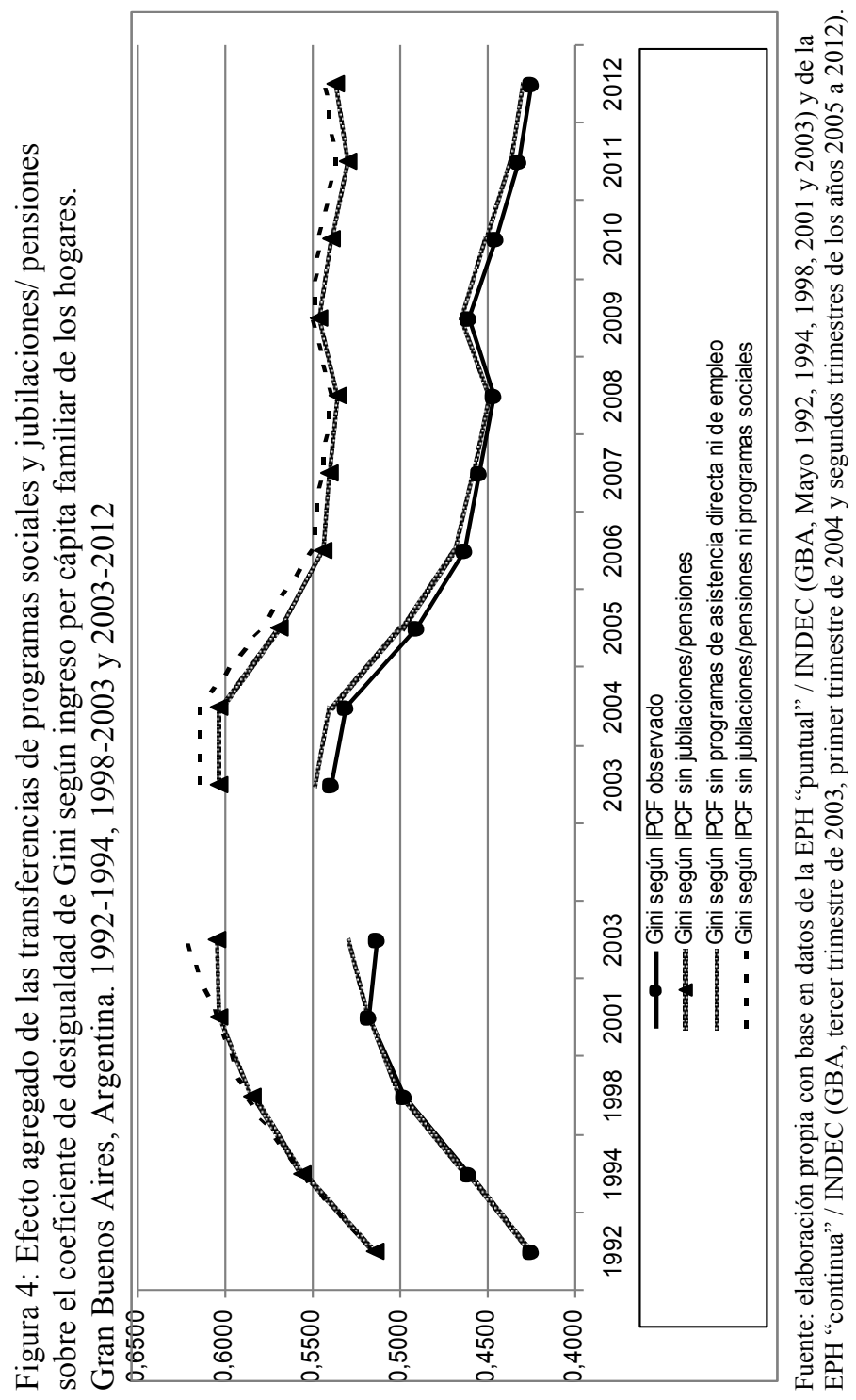


De este modo, es de esperar que el índice de desigualdad de Gini se haga más equitativo si tales transferencias y ayudas económicas producen una transferencia de renta de los hogares ubicados en la parte superior de la estratificación hacia las familias o personas ubicadas en las posiciones inferiores (principio de Pigou-Dalton). ${ }^{13}$

A partir de la información presentada, el análisis de cada período político-económico considerado da cuenta de los siguientes hallazgos:

1. En primer lugar, en la fase 1992-1998 se evidenció una primera etapa de crecimiento de los ingresos medios familiares entre 1992 y 1994, para luego pasar a una fase recesiva. En igual sentido, el índice de desigualdad de Gini presentó una evolución desfavorable, sólo en parte compensada por los ingresos generados por las jubilaciones y pensiones. Justamente, la exclusión de los ingresos por jubilaciones/ pensiones hace descender significativamente el promedio de ingresos de los hogares, más allá del año considerado. La brecha entre los ingresos totales y aquellos netos de jubilaciones/pensiones aumenta muy ligeramente entre los años 1992 y 1998, dando cuenta así de un muy leve incremento de la incidencia de la seguridad social en los ingresos promedio. Adicionalmente, se observa el impacto casi nulo que tuvieron los programas de asistencia social y de protección al desempleo (programas de protección contra la pobreza) en los ingresos medios durante todo el periodo analizado. Esto se debe a los magros montos otorgados por dichos programas, en comparación con las percepciones de los hogares originadas en el mercado laboral o fuera del mismo (a través de rentas, utilidades, etc.) La exclusión de los ingresos de la seguridad social hace incrementar la desigualdad distributiva, a lo largo del período, sin que se registren cambios a lo largo de la fase 19921998. A su vez, los programas de protección contra la pobreza casi no alteran la desigualdad durante el período 1992-1998.

2. Entre 1998 y 2003, en un contexto de fuerte deterioro de los indicadores socioeconómicos, los ingresos provenientes del sistema de seguridad social, tal como se mencionó anteriormente, contribuyeron a mejorar los indicadores en cada uno de los años analizados. Sin embargo, el grado de incidencia de los ingresos provenientes de jubilaciones o pensiones no cambió de manera relevante entre los años 1998 y 2003.

\footnotetext{
${ }^{13}$ Un buen indicador de desigualdad debe cumplir con la condición de Pigou-Dalton, es decir al realizar una transferencia de ingresos de una persona rica a una persona pobre se debe reducir el valor del indicador, en tanto que una transferencia de una persona pobre a una rica debe acrecentarlo.
} 
En estos años, los programas de asistencia directa y de protección al desempleo no lograron mejorar los ingresos de los hogares. Sin embargo, los programas sociales sí habrían logrado amortiguar el aumento de la desigualdad distributiva en el contexto de la crisis económicofinanciera 2001-2002. ${ }^{14} \mathrm{Si}$ bien el efecto compensatorio de los programas sobre la desigualdad es el más relevante del período estudiado, aún en esta fase los programas sociales sólo constituyeron un "paliativo" limitado para lograr niveles más equitativos de distribución. Es decir, no tuvieron efectos permanentes ni estructurales en la configuración de la estructura social. ${ }^{15}$

3. Entre 2003 y 2007, el ingreso medio y la desigualdad mejoraron de manera sostenida. Ahora bien, cabe preguntarse en qué medida la intervención estatal, a través del sistema de seguridad social y los programas de asistencia social directa y de protección al desempleo, alteraron los niveles y las tendencias de dichos indicadores. $\mathrm{Al}$ respecto, se observa que al excluir los ingresos de la seguridad social, la concentración de Gini es significativamente superior. La evolución de la brecha de estos indicadores estimados con los ingresos observados y los ingresos netos generados por jubilaciones/pensiones estaría indicando un efecto favorable, mucho mayor de la seguridad social conforme fue avanzando el periodo 2003-2007. ${ }^{16}$ Esto daría cuenta que a lo largo del periodo de políticas "heterodoxas" los ingresos de la seguridad social fueron contribuyendo de una manera cada vez más efectiva al alcance de niveles más equitativos de distribución de los ingresos.

4. Durante la última fase (2007-2012), la incidencia de los programas de protección contra la pobreza en los niveles de ingreso y desigualdad no se alteró significativamente. No sucede lo mismo con los ingresos de la seguridad social, los cuales sí parecen aumentar su impacto en los indicadores socioeconómicos seleccionados a lo largo de estos años. En un

${ }^{14}$ Esto se evidencia a través del incremento de la brecha — durante 1998-2003- entre los niveles de desigualdad que emplean los ingresos totales y aquellos estimados sobre el ingreso neto de programas sociales. Es decir, la exclusión de los ingresos por programas sociales hace aumentar muy levemente la desigualdad, siendo mayor este efecto en los años cercanos a la crisis de la convertibilidad —en pleno auge del Programa Plan Jefas y Jefes de Hogar-.

${ }^{15}$ Tal como se ha puesto en evidencia en diferentes trabajos (Galasso y Ravallion, 2003; Cortés et al., 2003; Monza y Giacometti, 2003; Bogani et al., 2005) la intervención pública a través de los programas de protección contra el desempleo — en particular el PJHD — habría logrado amortiguar el aumento de la pobreza y de la desigualdad de ingresos que generó la crisis económica. ${ }^{16}$ La evolución de la brecha a la cual acá se hace referencia queda representada a través del aumento de la distancia o el acercamiento entre las curvas que incluyen y excluyen los ingresos provenientes de jubilaciones y pensiones. El apartado siguiente pretenden dar una idea más acabada y detallada acerca de los cambios en la desigualdad durante distintas fases políticoeconómicas y el papel del gasto social (jubilaciones/pensiones y programas sociales) en dichos cambios. 
contexto de crecimiento económico más moderado y aceleración del proceso inflacionario, continuaron y se profundizaron los cambios que ya se habían iniciado - a partir de 2005- en el régimen de previsión social orientados a la recomposición del poder adquisitivo del beneficio previsional y la mejora en el alcance y nivel de las prestaciones. Estos cambios en el sistema de seguridad social incidieron en el papel que adquirió este tipo de beneficios sobre las tendencias de los indicadores de ingresos y distribución. Entre 2007 y 2012 se incrementó el impacto positivo de los ingresos provenientes de la seguridad social sobre dichos indicadores.

\section{EFECTO DE LA POLÍTICA SOCIAL Y SUS INGRESOS GENERADOS EN LOS MERCADOS SOBRE LOS CAMBIOS REGISTRADOS EN EL ÍNDICE DE GINI}

¿En qué medida y en qué sentido las transferencias de ingresos provenientes de la seguridad social y de los programas sociales de protección contra la pobreza - sean de protección contra el desempleo o de asistencia directa - han incidido en el nivel y en la evolución de la desigualdad en la distribución del ingreso entre los hogares del Gran Buenos Aires a lo largo del período estudiado? ¿En qué medida la mayor o menor focalización de los programas sociales se tradujo en una distribución más igualitaria? Con el objetivo de atender estas interrogantes resulta relevante retomar el método de descomposición del coeficiente de Gini desarrollado por Leibbrandt et al. (1996). ${ }^{17}$ Para una descripción detallada acerca de la descomposición del coeficiente de Gini, así como también de los factores/componentes involucrados en la misma ( $R, G$ y S), véase el apartado metodológico.

El presente apartado exhibe y analiza los resultados de la aplicación del modelo de descomposición buscando dar cuenta de la composición de la desigualdad, y a su vez, de los cambios ocurridos en el coeficiente de desigualdad de Gini de ingresos familiares en el Gran Buenos Aires. La Tabla 1 exhibe la capacidad de las transferencias directas de ingreso en materia de políticas sociales - a través de las jubilaciones/pensiones y programas sociales - para incidir en el patrón de distribución. Los datos dan cuenta de la contribución de estas fuentes de ingresos al índice de desigualdad de Gini. La Tabla 2 introduce un análisis dinámico de la desigualdad, exhibiendo las variaciones de los aportes al Gini por parte de cada una de las fuentes. Por último, la Tabla 3 presenta los factores/componentes $(R, G y S)$

\footnotetext{
${ }^{17}$ Siguiendo este modelo, Cortés (2000) analiza el papel de las diferentes fuentes de ingreso en la evolución de la desigualdad en México; y Medina y Galván (2008) lo desarrollan para distintos países de América Latina. A nivel nacional, Trujillo y Villafañe (2011), Salvia (2012) y Salvia y Vera (2013) retoman esta metodología.
} 
que subyacen a los cambios en el aporte que una determinada fuente hace a la desigualdad general. En el anexo se pueden consultar los resultados arrojados por la descomposición del Gini para cada año según tipo de ingreso y componente $(R, G y S)$.

Tabla 1: Aportes de las fuentes de ingreso seleccionadas al coeficiente de Gini. Gran Buenos Aires, Argentina. Años seleccionados

\begin{tabular}{lrrrrrr}
\hline & 1992 & 1998 & 2003 & $2003(3 \mathrm{er} \mathrm{T})$ & 2007 & 2012 \\
\hline $\begin{array}{l}\text { ITF / Neto de } \\
\text { transferencias }\end{array}$ & 0.4206 & 0.4460 & 0.4619 & 0.4685 & 0.4187 & 0.3845 \\
$\begin{array}{l}\text { Jubilaciones o } \\
\text { pensiones }\end{array}$ & -0.0049 & 0.0172 & 0.0267 & 0.0362 & 0.0055 & 0.0104 \\
$\begin{array}{l}\text { Programas sociales } \\
\begin{array}{l}\text { Programas de } \\
\text { empleo }\end{array}\end{array}$ & 0.0018 & 0.0001 & -0.0058 & -0.0037 & -0.0020 & -0.0012 \\
$\begin{array}{l}\text { Programas de } \\
\begin{array}{l}\text { asistencia directa } \\
\text { Total Gini ITF }\end{array}\end{array}$ & 0.0000 & -0.0005 & -0.0067 & -0.0032 & -0.0004 & 0.0000 \\
\hline
\end{tabular}

Fuente: elaboración propia con base en datos de la EPH "puntual" / INDEC (GBA, Mayo 1992, 1998 y 2003) y de la EPH "continua" / INDEC (GBA, tercer trimestre de 2003, y segundos trimestres de los años 2007 y 2012).

Tabla 2: Aportes de las fuentes de ingreso seleccionadas a la variación del coeficiente de Gini. Gran Buenos Aires, Argentina. Periodos seleccionados

\begin{tabular}{lrrrrrrrr}
\hline & \multicolumn{2}{c}{ VAR 1992-1998 } & \multicolumn{2}{r}{ VAR 1998-2003 } & \multicolumn{2}{r}{ VAR 2003-2007 } & \multicolumn{2}{r}{ VAR 2007-2012 } \\
& Abs & $\%$ & Abs & $\%$ & Abs & $\%$ & Abs & $\%$ \\
\hline ITF / Neto de transferencias & 0.0254 & 55.5 & 0.0159 & 81.6 & -0.0498 & 63.1 & -0.0342 & 119.9 \\
Jubilaciones o pensiones & 0.0220 & 48.1 & 0.0095 & 48.7 & -0.0308 & 39.0 & 0.0049 & -17.3 \\
Programas sociales & -0.0017 & -3.7 & -0.0059 & -30.0 & 0.0018 & -2.2 & 0.0007 & -2.6 \\
Programas de empleo & -0.0005 & -1.1 & -0.0063 & -32.2 & 0.0028 & -3.6 & 0.0004 & -1.4 \\
Programas de asistencia directa & -0.0012 & -2.7 & 0.0004 & 2.2 & -0.0011 & 1.4 & 0.0003 & -1.1 \\
Total Gini ITF & 0.0458 & 100.0 & 0.0195 & 100.0 & -0.0788 & 100.0 & -0.0285 & 100.0 \\
\hline Fuente: elaboración propia con base en datos de la EPH "puntual" / INDEC (GBA, Mayo 1992, 1998 y 2003) y de \\
la EPH "continua" / INDEC (GBA, tercer trimestre de 2003, y segundos trimestres de los años 2007 y 2012).
\end{tabular}




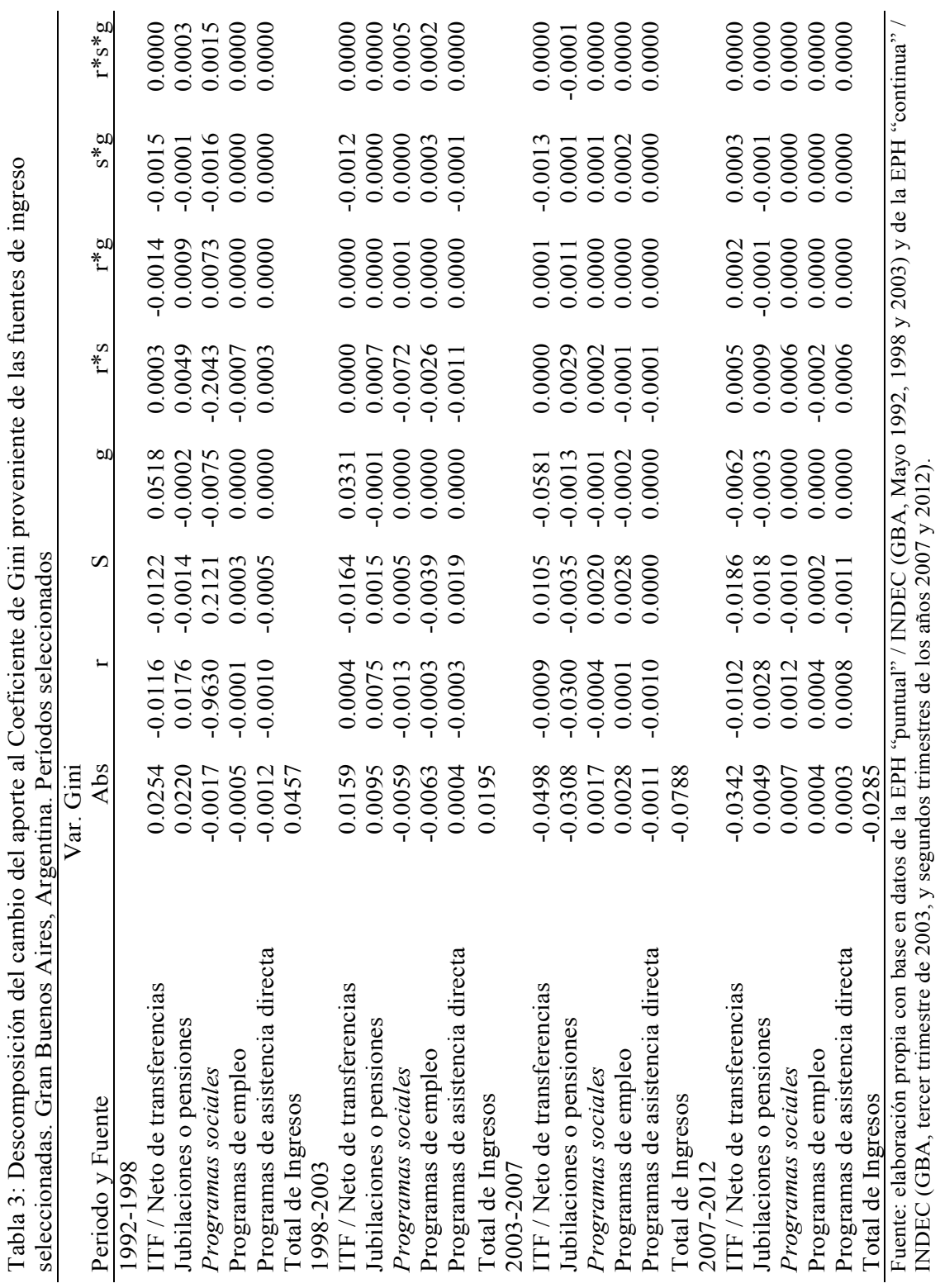


A continuación se destacan los hallazgos más relevantes para cada uno de los períodos político-económicos objeto de análisis:

1) En primer lugar, se observa que si bien en 1992 las transferencias a través de jubilaciones y pensiones compensaban las desigualdades originadas por otras fuentes de ingreso, muy rápidamente esta situación se modificó en los años posteriores (1992-1998): los ingresos por la seguridad social obtenidos por los hogares se convirtieron en una fuente cada vez más generadora de desigualdades a lo largo del período neoliberal (Tabla 1).

$\mathrm{Al}$ respecto, cabe destacar que desde el punto de vista institucional, una de las transformaciones más importantes del modelo neoliberal fue la fragmentación del sistema previsional a partir de la generación de un sistema privado de capitalización que se instaló junto con el clásico sistema público de reparto (Vera y Poy, 2017) En este contexto, se evidenciaron dos procesos simultáneos: una sistemática caída de la cobertura previsional de la población mayor de 65 años y un aumento de la desigualdad interna de las transferencias jubilatorias como resultado del deterioro de la prestación estatal (Vera y Poy, 2017). A su vez, el pasaje al sistema de capitalización benefició a los perceptores mejor posicionados frente a los del primer y segundo quintil, perjudicados por el deterioro del llamado sistema de reparto (Arza, 2010). Estos procesos habrían explicado la contribución de la previsión social a la inequidad observada.

Por otra parte, durante el mismo período, la primera generación de programas sociales habrían amortiguado sólo ligeramente la tendencia al crecimiento de la desigualdad provocado por otras fuentes (incluidas las jubilaciones/pensiones). Entre 1992 y 1998 el aumento de la desigualdad respondió en 48 por ciento a lo ocurrido con los ingresos de la seguridad social (Tabla 2). Esto es, las jubilaciones y pensiones regeneraron o intensificaron una estructura regresiva de distribución de ingresos. Esto se debió - íntegramente - a un aumento de la correlación de estos ingresos con la distribución general (Tabla 3). Es decir, durante una fase en la que primaron los procesos de reformas estructurales, a la vez que la generación de recursos quedó cada vez más en correspondencia con el "libre" funcionamiento de los mercados, la percepción de haberes previsionales prevaleció en aquellos hogares mejor posicionados en la estructura distributiva.

2) El aumento del aporte de los ingresos de la seguridad social al Gini continuó entre 1998 y 2003. Tal como se señaló anteriormente, la contribución de la seguridad social a una reproducción de las desigualdades existentes se explicó, fundamentalmente, por el incremento de la correla- 
ción de estos ingresos con la distribución general. ${ }^{18}$ Estos resultados adquieren sentido en el marco de una intensificación de la heterogeneidad de la pirámide jubilatoria y retracción en los niveles de cobertura, dando continuidad al proceso ya mencionado en la fase previa. ${ }^{19}$ Adicionalmente, cabe destacar que durante la fase recesiva y de crisis económica 1998-2003 $-\mathrm{y}$ en un contexto de implementación del Plan Jefas y Jefes de Hogar Desocupados - fueron los planes de protección contra el desempleo los que amortiguaron de manera importante las desigualdades generadas por las condiciones generales de los mercados. Dado que los planes de empleo contribuyeron a un menor nivel de desigualdad (tanto en 1998 como en 2003), el incremento de la participación de estos ingresos en la masa total de la economía ayudó a reducir de manera relevante su aporte al Gini, lo cual se vio reflejado en el comportamiento del componente $\mathrm{S}$-participación-(Tabla 3). También por el efecto de interacción entre S y R; es decir, no sólo se incrementó la participación de estos programas en la masa total de ingresos -lo cual hizo que descienda el Gini por ese exclusivo hecho-, sino también que este crecimiento fue más intenso en los hogares más desfavorecidos de la estructura social por vía de la focalización.

Tal como se ha puesto en evidencia en diferentes trabajos (Galasso y Ravallion, 2003; Cortés, Groisman y Hoswoszki, 2003; Monza y Giacometti, 2003; Bogani et al., 2005) y en línea con los resultados aquí obtenidos, la intervención estatal habría logrado amortiguar el aumento de la pobreza y de la desigualdad de ingresos que generó la crisis del programa económico. De no haber existido estas transferencias de ingresos - y suponiendo que los perceptores de los programas no hubiesen desarrollado otra actividad económica - los hogares de menores recursos hubieran visto afectada más seriamente su deteriorada capacidad de consumo.

3) Entre los años 2003 y 2007, la mejora en la distribución de los ingresos se originó - 40 por ciento aproximadamente - por lo ocurrido con las transferencias generadas por jubilaciones y pensiones. Si bien los ingresos de la seguridad social continuaron generando desigualdades, esto sucedió cada vez con menor intensidad durante el período post reformas estructurales. Esto se explicó fundamentalmente por una disminución de la correlación de las jubilaciones y pensiones con la distribución general: los estratos más desfavorecidos lograron un mayor acceso a este tipo de

\footnotetext{
${ }^{18}$ También entre 1998 y 2003, la percepción de haberes previsionales se intensificó en aquellos hogares mejor posicionados en la estructura distributiva.

${ }_{19}$ Para una evaluación detallada y sistemática acerca de los cambios y tendencias ocurridas en el sistema previsional argentino durante las últimas décadas, véase, entre otros, Arza (2010) y Curcio y Beccaria (2013).
} 
ingresos durante el período de políticas "heterodoxas". Según Danani y Beccaria (2011), durante el período de políticas "heterodoxas" tuvo lugar un proceso de contra-reforma del sistema previsional. Según las autoras, se trataría así de un sistema que ahora protege más y mejor. La expansión en el sistema previsional se implementó a través de dos iniciativas que contrastaron con períodos anteriores: un aumento significativo de la cobertura previsional y una recuperación de los haberes, en especial de la parte más baja de la pirámide previsional (Vera y Poy, 2017). Así, las "contra-reformas" en materia de programas sociales y seguridad social habrían tenido un efecto positivo sobre la reducción de la desigualdad. Diversos estudios destacan el papel central del sistema de jubilaciones y pensiones, que durante el ciclo heterodoxo incrementó su cobertura y redujo su desigualdad interna, incidiendo positivamente sobre la desigualdad (Trujillo y Villafañe, 2011; Cruces y Gasparini, 2009).

A su vez, en este periodo los programas sociales continuaron siendo fuentes de ingreso que compensaron desigualdades generadas por otras fuentes. Sin embargo, cabe señalar algunas rupturas respecto a la década de reformas estructurales. En primer lugar, debido a que la función compensadora - aunque limitada - ejercida por los programas de protección contra el desempleo inmediatamente luego de la crisis de la devaluación (Salvia y Vera, 2013) estuvo prácticamente ausente durante la fase $2003-2007 .{ }^{20} \mathrm{De}$ manera inversa, la contribución de las transferencias no laborales (programas de asistencia directa) al Gini se hizo más negativa ente los años 2003 y 2007; es decir, que este tipo de transferencias aportaron — cada vez con mayor intensidad - a descender los niveles de desigualdad existentes. Esto encuentra explicación en la disminución de la correlación de este tipo de ingresos con la distribución general, revelando que cada vez más correlación aquellos hogares que percibían transferencias por programas sociales continuaban siendo los más desfavorecidos en la estructura social. Los resultados empíricos obtenidos se explican en el marco de una activa política gubernamental en materia de ingresos a partir del año 2003: mejora en el monto de los haberes previsionales y ampliación de la cobertura, avances en el salario mínimo, vital y móvil, aumentos salariales a los trabajadores del sector privado, incremento de las negociaciones colectivas, transferencias de ingresos, y significativo incremento de pensiones no contributivas

\footnotetext{
${ }^{20}$ Durante la fase de consolidación de políticas "heterodoxas" los planes sociales de empleo van perdiendo la relevancia que tenían anteriormente, ganando - en contrapartida - participación los programas sociales de transferencias monetarias. Dado que los planes de empleo contribuyen compensando las desigualdades generadas por otras fuentes, la pérdida de relevancia de este tipo de ingresos en la economía (entre 2003 y 2007) hace incrementar el aporte de esta fuente al Gini vía el componente $\mathrm{S}$ - participación en el volumen de ingresos-
} 
más ligadas a la seguridad social. Durante el período de políticas heterodoxas ganaron preponderancia las transferencias condicionadas de ingresos, contributivas y no contributivas, disminuyendo así la relevancia de los programas de empleo.

4) En la última fase del período analizado (2007-2012) la mejora en la distribución de los ingresos no se habría explicado por ninguna de las fuentes particulares analizadas. Tanto las transferencias por jubilaciones y pensiones como los programas sociales de protección contra la pobreza incrementaron su aporte al Gini entre estos años. Si bien los programas de asistencia directa tendieron a compensar las desigualdades generadas por el funcionamiento del mercado, el cambio en este tipo de ingresos entre 2007 y 2012 no fue relevante. En el balance 2007-2012 quedarían invisibilizadas estas tendencias disímiles pre y post implementación de la AUH (a fines del 2009). De esa manera, la disminución de la desigualdad en el Gran Buenos Aires durante el último período podría entenderse por la menor disparidad del ingreso laboral, originada por factores como la desvalorización del "premio" a la educación, la caída del desempleo, los incrementos de suma fija y la recomposición del salario mínimo, medidas que impactan con mayor intensidad en los estratos de ingresos más bajos.

La literatura especializada destaca el papel que jugó la demanda de empleo en la mejora distributiva en los años 2000. En el caso argentino, la sustitución de importaciones permitió una demanda de empleo más vigorosa y con elevada participación de ocupados no calificados o semi-calificados (Cruces y Gasparini, 2009). El cambio de precios relativos posterior a la devaluación favoreció a las empresas intensivas en trabajo no calificado, que habían enfrentado una dura competencia en la liberalización, por lo que el crecimiento de la actividad industrial mejoró los ingresos de los menos calificados (Kessler, 2014). Como resultado, se habrían reducido - a lo largo de esta fase - las "primas" a la educación también como resultado del mayor capital educativo de la fuerza de trabajo. En línea con estas reflexiones, la CEPAL (2013) señala que la disminución de la desigualdad en la Argentina se explica principalmente por una menor disparidad del ingreso laboral, originada por factores como la desvalorización del "premio" a la educación, la caída del desempleo y el alza del ingreso mínimo, entre otros. En este contexto, los cambios en las políticas sociales habrían jugado un papel importante, pero secundario con respecto a los cambios verificados en la dinámica de los mercados de trabajo. 


\section{REFLEXIONES FINALES}

Este trabajo se ha propuesto analizar el impacto específico de las políticas sociales - tomando en consideración los programas de asistencia social directa, los planes de protección al desempleo y las transferencias previsionales (jubilaciones y pensiones) — sobre la desigualdad de los ingresos en la región del Gran Buenos Aires, Argentina. Se tomaron en cuenta dos fases de reglas macroeconómicas muy diferentes: la etapa de reformas estructurales (1992-2003) y la etapa "heterodoxa" o post-reformas (20032012).

Se destacó la efectiva vigencia de un cambio cualitativo entre la política social del período neoliberal y la correspondiente a la etapa pos-reformas estructurales. A diferencia de los programas focalizados de la década de los noventa, los programas de protección contra la pobreza en la etapa pos-reformas fueron adoptando un carácter más extendido, a la vez que las transferencias de la seguridad social (sistema de jubilaciones y pensiones) adquirieron una relevancia creciente en el presupuesto de los hogares. Esto ocurrió en un contexto en el cual también mejoró la distribución primaria (es decir, la que se origina en las relaciones sociales del trabajo).

Se aplicó un ejercicio de descomposición del coeficiente de Gini para cuantificar el aporte de cada una de estas fuentes a la desigualdad. Durante la década neoliberal, en el marco del proceso de privatización del sistema de la seguridad social, las jubilaciones y pensiones aportaron a una mayor desigualdad; mientras que los programas de lucha contra la pobreza operaron en sentido contrario. En un contexto de deterioro generalizado como de los ingreso, como fue la crisis 2001-2002, ambas transferencias operaron amortiguando el aumento de la desigualdad. Durante los primeros años de la década pos-reformas, las transferencias de la seguridad social habrían coadyuvado en la reducción de la desigualdad, al igual que los programas de asistencia directa.

La evidencia presentada permitió dar cuenta de los límites que enfrentan las políticas sociales "redistributivas" para resolver por sí solas las desigualdades estructurales que presenta el sistema productivo y el mercado de trabajo. Ello ha sido así tanto en un contexto de crecimiento del empleo y mejora de los ingresos laborales de los sectores formales, como en un contexto regresivo en la distribución del ingreso a nivel general. Más allá de las diferencias sustantivas que han presentado los programas macro-económicos de diferente signo aquí comparados, las políticas sociales aplicadas en la Argentina durante las últimas dos décadas parecen 
haber funcionado apenas como mecanismos redistributivos "compensadores" de exclusiones estructurales o coyunturales que genera la dinámica dominante de acumulación. la cual mantiene una elevada especialización y concentración económica, al mismo tiempo que una fuerte dependencia hacia el mercado mundial. De este modo, se ha perdido de vista o se ha dejado en un segundo plano, el problema de la efectiva integración social sistémica que requiere un programa de desarrollo orientado a una mayor convergencia social.

Desde esta perspectiva, parecen ser necesarias transformaciones más significativas en el sistema económico y productivo que se trasladen a la configuración del mercado de trabajo, de manera tal de mejorar las condiciones de los empleos ofrecidos y las oportunidades de acceso a los mismos. Es así que encontrar el camino del desarrollo significa repensar la estructura del sistema económico y el mercado de trabajo generado bajo dicho sistema, dado que son estos los factores que determinan y condicionan — en mayor parte— los procesos de desigualdad socioeconómica.

\section{REFERENCIAS BIBLIOGRÁFICAS}

Arza, Camila, 2010, "La política previsional: de la estratificación ocupacional a la individualización de los beneficios", en Torrado, S. (dir.) El costo social del ajuste. Argentina (1976-2002), T. 2, pp. 257-300, Buenos Aires: EDHASA.

Barba Solano, Carlos y Cohen, Néstor, 2011, Perspectivas críticas sobre la cohesión social, Buenos Aires: CLACSO.

Barrientos, Armando y Hulme, David, 2008, Social protection for the poor and poorest in developing countries: reflections on a quiet revolution, BWPI Working Paper, Manchester.

Bertranou, Fabio y Paz, Jorge, 2007, Políticas y programas de protección al desempleo en Argentina, Buenos Aires: OIT.

Birdsall, Nancy y De la Torre, Augusto, 2001, El Disenso de Washington. Políticas económicas para la equidad social en Latinoamérica. Fondo Carnegie para la Paz Internacional y Diálogo Interamericano.

Bogani, Esteban; Grosso, Marco; Philipp, Ernesto; Salvia, Agustín y Zelarayan, Julio, 2005, "Aunque no alcance, se trata de sumar. El comportamiento de las mujeres, varones y hogares en el Plan Jefes y Jefas de Hogar Desocupados", en Revista Argentina de Sociología, CPS, año 3, núm. 5, noviembre-diciembre de 2005. Págs 187-205.

Bonvecchi, Alejandro y Smulovitz, Catalina, 2008, "Atender necesidades, crear oportunidades o garantizar derechos. visiones sobre la política social", en Cruces, G., Ringold, D. y Rofman, R. (eds), Los programas sociales en Argentina hacia el Bicentenario. Visiones y perspectivas, Banco Mundial, Oficina de Buenos Aires. 
CELS, 2009, Presentación de recurso de reconsideración con recurso jerárquico en subsidio. Solicitan medidas. Centro de Estudios Legales y Sociales Recuperado de http://www.cels.org.ar/common/documentos/INDEC_recurso.pdf (consulta: 10/05/2014).

CEPAL, 2009, Panorama social de América Latina, Santiago de Chile.

CEPAL, 2013, Panorama social de América Latina, Santiago de Chile.

CEPAL, 2014, Pactos para la igualdad. Hacia un futuro sostenible, Santiago de Chile.

CIFRA, 2011, El nuevo patrón de crecimiento. Argentina 2002-2010. Informe de Coyuntura núm. 7. Centro de Investigación y Formación de la República Argentina-CIFRA.

Cortés, Fernando, 2000, La distribución de la riqueza en México en épocas de estabilización y reformas económicas. Centro de Investigaciones y Estudios superiores en Antropología Social, México: M. A. Porrua Grupo Editorial.

Cortés, Fernando, 2013, "Medio siglo de desigualdad en el ingreso en México", en Economía UNAM, vol. 10. Facultad de Economía UNAM, pp. 136.

Cortés, Fernando, 2014, Gasto social y pobreza, Documento de Trabajo núm. 9, Programa Universitario de Estudios del Desarrollo, Universidad Nacional Autónoma de México.

Cortés, Rosalía, Groisman, Fernando y Hoswoszki, Augusto, 2003, Transiciones ocupacionales: el caso del Plan Jefes y Jefas, ponencia presentada en el $6^{\circ}$ Congreso Nacional de Estudios del Trabajo de la Asociación Argentina de Especialistas en Estadios del Trabajo. ASET, Buenos Aires.

Cruces, Guillermo y Gasparini, Leonardo, 2009, Los determinantes de los cambios en la desigualdad de ingresos en la Argentina. Evidencias y temas pendientes, Serie Documentos de Trabajo sobre políticas sociales, núm. 5, Banco Mundial.

Curcio, Javier y Beccaria, Alejandra, 2013, "Sistema de Seguridad Social y mercado de trabajo. Evolución de la cobertura en la Argentina entre 1990 y 2010", en Danani, C. y Hintze, S. (coords.) Protecciones y desprotecciones: la seguridad social en la Argentina (1990-2010), T. 1, pp. 61-102, Los Polvorines: UNGS.

Damill, Mario y Frenkel, Roberto, 2006, "El mercado de trabajo argentino en la globalización financiera”, en Revista CEPAL, núm. 88, 109-132.

Damill, Mario, Frenkel, Roberto y Maurizio, Roxana, 2011, Macroeconomic policy for full and productive employment and decent work for all: An analysis of the Argentine experience, Employment Working Paper, OIT.

Danani, Claudia y Beccaria, Alejandra, 2011, "La (contra)reforma previsional argentina 2004-2008: aspectos institucionales y político-culturales del proceso de transformación de la protección", enn Danani, C y S. Hintze (coords), Protecciones y desprotecciones. La seguridad social en la Argentina 1990-2010. Págs. 103151. Universidad Nacional de General Sarmiento. Colección Política, políticas y sociedad. 
Danani, Claudia y Grassi, Estela, 2008, "Ni error ni omisión. El papel de la política de estado en la producción de las condiciones de vida y de trabajo", enn Lindenboim J. (comp.) Trabajo, ingresos y politicas en Argentina. Contribuciones para pensar el siglo XXI. Buenos Aires, EUDEBA.

Danani, Claudia y Hintze, Susana, 2011, "Introducción. Protección y seguridad social para distintas categorías de trabajadores: definiciones conceptuales, propuestas de abordaje e intento de interpretación", en Danani, C y S. Hintze (coords), Protecciones y desprotecciones. La seguridad social en la Argentina 1990-2010. Los Polvorines: Universidad Nacional de General Sarmiento.

De Ferranti, David; Perry, Guillermo; Ferreira, Francisco y Walton, Michael, 2003, Desigualdad en América Latina y el Caribe: ¿ruptura con la historia? Banco Mundial, Washington, D.C.

Falappa, Fernando y Andrenacci, Luciano, 2008, La política social de la Argentina democrática (1983-2008), Buenos Aires: Biblioteca Nacional.

Ferreira, Francisco; Messina, Julián; Rigolini, Jamele; López-Calva, Luis; Lugo, María y Vakis, Renos, 2013, La movilidad económica y el crecimiento de la clase media en América Latina. Washington, DC: Banco Mundial.

Galasso, Emanuela y Ravallion, Martín, 2003, Social Protection in a Crisis: Argentina's Plan Jefes y Jefas. Development Research Group, World Bank. Washington DC.

Gasparini, Leonardo, Cruces, Guillermo y Tornarolli, Leopoldo, 2011, "Recent Trends in Income Inequality in Latin America", en Economía, vol. 10 (2), pp. 147201, Spring, 2011.

Grassi, Estela, 2003, Políticas y problemas sociales en la sociedad neoliberal. La otra década infame (I). Buenos Aires: Editorial Espacio.

Grassi, Estela, Hintze, Susana y Neufeld, Rosa, 1994, Políticas sociales, crisis y ajuste estructural. Buenos Aires: Espacio Editorial.

Hernández Laos, Enrique, 2006, Mercado laboral, desigualdad y pobreza en América Latina, México: Porrúa.

INDEC, 2016, "Bases Usuarias" correspondientes a la Región Gran Buenos Aires: ondas mayo de 1992, 1998 y 2003 (EPH "puntual") y el tercer trimestre de 2003, primer trimestre de 2004 y segundos trimestres de los años 2005 a 2012 (EPH "continua"). Dirección de Encuesta Permanente de Hogares (EPH)

INDEC, 2009, "Ponderación de la muestra y tratamiento de valores faltantes en las variables de ingreso en la EPH", en Metodología núm. 15, INDEC, Buenos Aires Isuani, Aldo, 2008, "La política social argentina en perspectiva", en Cruces, G., D. Ringold y R. Rofman (eds), Los programas sociales en Argentina hacia el Bicentenario. Visiones y perspectivas, Banco Mundial, Oficina de Buenos Aires.

Kaztman, Ruben y Wormald, Guillermo, 2002, Trabajo y Ciudadanía. Integración y Exclusión Social en cuatro áreas metropolitanas de América Latina, Montevideo: Cebra. 
Kessler, Gabriel, 2014, Controversias sobre la desigualdad. Argentina, 20032013. Fondo de Cultura Económica.

Leibbrandt, Murray; Woolard, Christopher y Woolard, Ingrid, 1996, The contribution of income components to income inequality in South Africa: A decomposable Gini Analysis. LSMS Working Paper núm. 125. Washington: The World Bank.

Levinas, 2014, "Políticas socials en América Latina en el siglo XXI. Los programas de transferencias monetarias condicionadas", en Revista Desarrollo Económico, 54(212), 3-34.

Lindenboim, Javier, 2012, "La pobreza: una tensión social más allá de la metrópolis", en Ainstein, Luis (compilador) Estructuración urbana, institucionalidad y sustentabilidad de ciudades metropolitanas y regiones difusas. Miradas comparadas sobre Buenos Aires, Londres, Los Ángeles, Paris, Tokio y Toronto. Buenos Aires: Eudeba.

Lo Vuolo, Rubén, y Barbeito, Alberto, 1998, La nueva oscuridad de la política social. Del Estado Populista al Neoconservador. (Eds.), Miño y Dávila Editores - Ciepp. Buenos Aires.

Lustig, Nora; López-Calva, Luis; Ortiz-Juarez, Eduardo, 2011, The decline in inequality in Latin America: How much, since when and why. ECINEQ WP 2011211.

Medina, Fernando y Galván, Marco, 2008, Descomposición del coeficiente de Gini por fuentes de ingreso. Evidencia empírica para América Latina (1999-2005). Serie de estudios económicos y prospectivos. Santiago de Chile, CEPAL.

Medina, Fernando y Galván, Marco, 2014, Crecimiento económico, pobreza y distribución del ingreso. Fundamentos teóricos y evidencia empírica para América Latina, 1997-2007. Serie Estudios Estadísticos. Santiago de Chile, CEPAL.

Monza, Alfredo y Giacometti, Claudia, 2003, Los beneficiarios del Plan Jefas y Jefes de Hogar. Enfrentando los retos al trabajo decente en la crisis argentina. Proyecto de Cooperación Técnica OIT/Gobierno Argentino (MTEySS). Buenos Aires. (mimeo)

Neffa, Julio César y Panigo, Demian, 2009, El mercado de trabajo argentino en el nuevo modelo de desarrollo, Documento de Trabajo, Dirección Nacional de Programación Macroeconómica/ Dirección de Modelos y Proyecciones, Ministerio de Economía y Finanzas Públicas.

Novick, Marta, 2006, “¿Emerge un nuevo modelo económico y social? El caso argentino 2003-2006”, en Revista Latinoamericana de Estudios del Trabajo. Año 11, núm. 18.

Nun, José, 1969, "Superpoblación relativa, ejército industrial de reserva y masa marginal”, en Revista Mexicana de Sociología, núm. 2.

Palomino, Héctor, 2007, "La instalación de un nuevo régimen de empleo en Argentina: de la precarización a la regulación”, en Revista Latinoamericana de Estudios del Trabajo. Año 12, núm. 19. 
Perry, Guillermo; Maloney, William; Arias, Omar; López, Humberto y Servén, Luis, 2006, Reducción de la pobreza y crecimiento: círculos virtuosos y círculos viciosos. Bogotá: Banco Mundial.

Pinto, Aníbal, 1973, "Heterogeneidad estructural y modelo de desarrollo reciente en América Latina”, en Inflación: raíces estructurales. México: Fondo de Cultura Económica.

Prebisch, Raúl, 1949, El desarrollo económico de América Latina y algunos de sus principales problemas, Santiago de Chile: Naciones Unidas.

Prebisch, Raúl, 1963, Hacia una dinámica del desarrollo latinoamericano, México: Fondo de Cultura Económica.

Repetto, Fabián y Andrenacci, Luciano, 2005, "Ciudadanía y capacidad estatal: dilemas presentes en la reconstrucción de la política social argentina", en Andrenacci, L. (comp.) Problemas de política social en la Argentina contemporánea. Buenos Aires: Prometeo.

Rodríguez, Octavio, 2006, El estructuralismo latinoamericano, México: Siglo Veintiuno Editores.

Salvia, Agustín, 2012, La trampa neoliberal. Un estudio sobre los cambios en la heterogeneidad estructural y la distribución del ingreso en la Argentina: 19902003. Buenos Aires: Eudeba.

Salvia, Agustín y Vera, Julieta, 2012, "Cambios en la estructura ocupacional y en el mercado de trabajo durante fases de distintas reglas macroeconómicas (19922010)", en Revista Estudios del Trabajo, núm. 41/42, ASET Buenos Aires.

Salvia, Agustín y Vera, Julieta, 2013, "Heterogeneidad estructural y desigualdad económica: Procesos intervinientes en el patrón de la distribución de los ingresos laborales del Gran Buenos Aires durante las distintas fases macroeconómicas (1992-2010)", en Revista Desarrollo Económico, núm. 207-208, vol. 52. Buenos Aires: Instituto de Desarrollo Económico y Social.

Salvia, Agustín, 2014, Heterogeneidades estructurales y desigualdades sociales persistentes. De la caída del modelo neoliberal a la falta de horizontes bajo el modelo neodesarrollista, Documento de Trabajo. ODSA-UCA: Buenos Aires.

Tokman, Víctor. y O'Donnell, Guillermo, 1999, Pobreza y desigualdad en América Latina, Buenos Aires, Paidós.

Trujillo, Lucía y Villafañe, Soledad, 2011, Dinámica Distributiva en la Argentina Reciente. Descomposición del Coeficiente de Gini por Fuentes de Ingreso, ponencia presentada en el $10^{\circ}$ Congreso Nacional de Estudios del Trabajo, Asociación Argentina de Especialistas en Estudios del Trabajo-ASET-. Buenos Aires, 3 al 5 de agosto de 2011.

Vera, Julieta y Poy, Santiago, 2017, "Mercado laboral, políticas sociales y desigualdad: cambios recientes en perspectiva histórica. Gran Buenos Aires, 19742014", en Revista Economía UNAM, vol. 14, núm. 42, septiembre-diciembre, 2017. pp 3-23. 


\section{RESUMEN CURRICULAR DE LOS AUTORES}

\section{Agustín Salvia}

Sociólogo y Magíster en Ciencias Políticas y Sociales de la Universidad Autónoma de México. Doctor en Ciencias Sociales de El Colegio de México. Investigador del CONICET. Profesor universitario en Metodología y Técnicas de Investigación en Ciencias Sociales a nivel de grado y de posgrado en la Universidad de Buenos Aires, la Universidad Nacional de 3 de Febrero, FLACSO Argentina y la Universidad de la República de Uruguay. Trabajos recientes: Salvia, Agustín, Robles, Ramiro y Fachal, María Noel (2017, en prensa) "Estructura sectorial del empleo, nivel educativo de la fuerza de trabajo y diferenciales de ingresos laborales en la Argentina (1992-2014)", en Cuadernos de Relaciones Laborales, ISSN: 1131-8635; Salvia, Agustín y Vera, Julieta (2016), "Calidad del empleo en Argentina (2004-2011). Una crítica al enfoque de las credenciales educativas", en Revista Ciencias Sociales (versión online). Facultad de Ciencias Sociales, Universidad de la República, Uruguay, vol. 29, núm. 38, ISSN 1688-4981; Salvia, Agustín; Poy, Santiago y Vera, Julieta (2016), "La política social y sus efectos sobre la pobreza durante distintas etapas macroeconómicas. argentina, 1992-2012”, en Revista Desarrollo y Sociedad, núm. 76, enerojunio, 2016, pp.165-203, Universidad de los Andes, Bogotá, Colombia. Dirección electrónica: agsalvia@retina.ar; agustin_salvia@uca.edu.ar

\section{Santiago Poy}

Lic. en Sociología (Universidad de Buenos Aires). Doctorando en Ciencias Sociales (Universidad de Buenos Aires). Becario doctoral del CONICET en el Programa del Observatorio de la Deuda Social Argentina (Universidad Católica Argentina). Docente de la carrera de Sociología de la Universidad de Buenos Aires. Trabajos recientes: Poy, S. (2017). "Cambios en la participación laboral de los hogares y en los niveles de bienestar económico. Argentina en los años post-reformas (2003-2014)", en Estudios del Trabajo, 51-52, s/p. ISSN 0327-5744; Vera, Julieta y Poy, Santiago (2017), "Mercado laboral, políticas sociales y desigualdad: cambios recientes en perspectiva histórica. Gran Buenos Aires, 1974-2014", en Revista Economía UNAM, vol. 14, núm. 42, septiembre-diciembre, 2017. pp 3-23. ISSN:1665-952x; Poy, S. (2017). "Heterogeneidad de la estructura ocupacional y segmentación del mercado de trabajo. Gran Buenos Aires, 1974-2014", en Trabajo y Sociedad, 29, pp. 353-376. ISSN 1514-6871. 
Dirección electrónica: santiagopoy@hotmail.com; santiago_poy@uca. edu.ar

\section{Julieta Vera}

Lic en Economía (Universidad de Buenos Aires) y Magíster en Diseño y Gestión de Políticas y Programas Sociales (FLACSO). Doctora en Ciencias Sociales (Universidad de Buenos Aires). Investigadora del Programa del Observatorio de la Deuda Social Argentina (Universidad Católica Argentina) y miembro del Programa Cambio Estructural y Desigualdad Social (IIGG-UBA). Trabajos recientes: Vera, Julieta y Poy, Santiago (2017), "Mercado laboral, políticas sociales y desigualdad: cambios recientes en perspectiva histórica. Gran Buenos Aires, 1974-2014", en Revista Economía UNAM, vol. 14, núm. 42, septiembre-diciembre, 2017. pp 3-23. ISSN:1665-952x; Salvia, Agustín y Vera, Julieta (2016), "Calidad del empleo en Argentina (2004-2011). Una crítica al enfoque de las credenciales educativas", en Revista Ciencias Sociales (versión online). Facultad de Ciencias Sociales, Universidad de la República, Uruguay. Vol.29, n.38, pp.37-58. ISSN 1688-4981. Vera, Julieta (2016), "Movilidad ocupacional en la Argentina en un contexto de heterogeneidad estructural", en Cuadernos del CENDES (versión online) vol. 32, núm. 90, Caracas. ISSN 2443$468 \mathrm{X}$

Dirección electrónica: julietavera@gmail.com; julieta_vera@uca.edu.ar 


\section{ANEXO METODOLÓGICO}

\section{Microdatos de la EPH del INDEC}

La utilización de micro datos de la EPH-INDEC requirió de procedimientos complementarios para hacer comparables los resultados. En general, la evaluación de la información se hizo tomando una serie de años ventana para el período 1992-2012 (1992, 1994, 1998, 2003, 2007, 2012). Pero a lo largo de estos periodos tuvieron lugar cambios de diverso orden en la encuesta de hogares. En lo que respecta a este trabajo, afectan especialmente los cambios en los ponderadores poblacionales, la ampliación de la cobertura geográfica y el modo de relevar las características ocupacionales y los ingresos. No obstante, el cambio más significativo tuvo lugar en el segundo semestre del año 2003, a partir del reemplazo de la antigua EPH "puntual" que tenía dos mediciones anuales, por la denominada encuesta EPH "continua" con cambio en cuatro tiempos de la estructura de rotación de la muestra.

Debido a los aspectos señalados, el análisis se limitó al área metropolitana del Gran Buenos Aires (GBA). Los años seleccionados corresponden a las ondas del mes de mayo de 1992, 1998 y 2003 (EPH "puntual") y al tercer trimestre de 2003, primer trimestre de 2004 y segundos trimestres de 2005 a 2012 (EPH "continua"). Dado que algunos de los ejercicios planteados en este trabajo contienen un nivel elevado de complejidad metodológica, se consideró que el empalme de datos entre la EPH "puntual" y "continua" podría resultar distorsivo y, por ende, menos fiable. Al mismo tiempo, la falta de información en los años 1992 y 1994 respecto a los ingresos por ayudas/subsidios no laborales obligó a trabajar en estos años con datos estimados (en base al peso que adquiere esta fuente en el total de ingresos no laborales en los años sucesivos). Por estos motivos, el análisis se desarrolló a través de una serie "cortada" de resultados: 1992-1994, 1994-1998, 1998-2003 y 2003-2012 (este último periodo, subdividido por razones teóricas en 2003-2007 y 2007-2012).

El sesgo de selección que arrastra la no declaración de los ingresos corrientes de los hogares obligó a llevar a cabo un tratamiento especial de los datos faltantes en esta dimensión. En este sentido, con el objetivo de disminuir la pérdida de información, así como del número de casos/hogares con algún miembro sin información, se tomó la decisión de estimar por tipo de fuente los ingresos personales no declarados — para los años seleccionados del periodo 1992-2003, es decir, para la modalidad EPH "puntual". Con esos fines se utilizó un modelo de regresión multivariado 
para la determinación de los ingresos por perceptor y tipo de ingreso. Los datos faltantes estimados fueron computados a los individuos y posteriormente agregados a los hogares.

En la modalidad EPH "continua" (2003-2012), a diferencia de la serie EPH "puntual”, el propio INDEC hace una imputación por registro y tipo de fuente de los ingresos no declarados. ${ }^{1}$ Este método de imputación de ingresos arroja resultados similares (estadísticamente no significativos) a los que se hubiesen obtenido a través del método de estimación propio empleado con las bases de la EPH "puntual". Por ese motivo, y también para simplificar las comparaciones con datos provenientes del organismo, se decidió aceptar el método de imputación de ingresos diseñado por el INDEC para las bases de la EPH “continua” (Anexo 1).

Anexo 1: Fuentes de datos y variables utilizadas para la construcción de indicadores de percepción de ingresos por transferencias de la seguridad social y programas de protección contra la pobreza

EPH Puntual (1992-1994)

Ingresos por Jubilaciones

Ingresos por Planes

Ingresos por Subsidios o ayudas p47_6 Otros ingresos

EPH Puntual (1998-2003)

Ingresos por Jubilaciones

Ingresos por Planes

Ingresos por Subsidios o ayudas

EPH Continua (2003-2012)

Ingresos por Jubilaciones

Ingresos por Planes

p48_1 Ingreso por jubilación o pensión

P48_1 Ingreso por jubilación o pensión

p18a Identifica Planes de empleo

p48_9 Otros ingresos

v2_m Monto percibido por jubilación o pensión

pp07e El trabajo es un Plan de Empleo

Ingresos por Subsidios o ayudas v5 $\mathrm{m}$ Monto percibido por subsidios o ayudas

Fuente: elaboración propia con base en cuestionarios de EPH "puntual" / INDEC (1992 a 2003) y EPH

"continua" / INDEC (2003 a 2012).

Para su adecuada comparación, los ingresos corrientes de los hogares se deflacionaron a precios de 2012, aplicando para ello el índice oficial de precios (IPC-GBA del INDEC) para el período 1992-2006, así como un índice elaborado y publicado por ex técnicos del INDEC (índice GB) para el periodo 2007-2012. Se sigue esta estrategia debido al hecho conocido en torno a la manipulación de los índices de precios que experimentó el IN-

\footnotetext{
${ }^{1}$ Para mayores especificaciones acerca de los cambios metodológicos que se introdujeron a partir de 2003 en las bases de datos de la EPH "continua" para el tratamiento de los valores faltantes en las variables de ingreso, véase el documento metodológico del organismo (INDEC, 2009).
} 
DEC a partir del año 2007 por parte de la Secretaría de Comercio Interior del Ministerio de Economía de la Nación. ${ }^{2}$

\section{Descomposición del Coeficiente de Gini}

El presente trabajo retoma el método de descomposición del coeficiente de Gini desarrollado por Leibbrandt et al. (1996), el cual permite especificar el aporte que realizan diferentes fuentes de ingreso en el nivel de desigualdad total, así como también los comportamientos subyacentes que intervinieron en los cambios del patrón distributivo. El aporte que una determinada fuente de ingreso $k$, en un tiempo $t$, haga a la desigualdad general habrá de depender no sólo de cuánto participe ese tipo de ingreso en el ingreso total $\left(\mathrm{S}_{\mathrm{k}, \mathrm{t}}\right)$, sino también de cuán desigual sea la distribución del ingreso de esa fuente $\left(\mathrm{G}_{\mathrm{k}, \mathrm{t}}\right), \mathrm{y}$, por último, cuánto el ingreso generado en esa fuente se correlaciona ordinalmente con la posición relativa del hogar en la distribución general $\left(\mathrm{R}_{\mathrm{k}, \mathrm{t}}\right){ }^{3}$

En el primer componente, obviamente, una concentración más alta / baja del ingreso en una fuente de ingreso habrá de favorecer una mayor / menor desigualdad en la distribución. En el segundo componente, cuanto menos / más equitativa sea la distribución del ingreso al interior de cada fuente, será mayor / menor el aporte que hará la fuente a la desigualdad general. Cuanta mayor correlación positiva / negativa exista entre los ingresos por parte de una fuente y la distribución general, la fuente habrá de aportar mucho más de manera regresiva /progresiva a la desigualdad.

Si se dispone de la información de las fuentes del ingreso monetario de los hogares a nivel de micro datos se confirma que:

$$
G=\sum_{k=1}^{K}\left(R_{k} G_{k} S_{k}\right)
$$

A partir de (1) y distinguiendo las fuentes de ingreso que son de interés aquí, el valor del coeficiente de Gini de ingresos de los hogares puede ser descompuesto de la siguiente forma:

$$
G_{t}=D_{\text {ITF(neto)t }}+D_{S S t}+D_{P S t} \text { donde: }
$$

${ }^{2}$ Los modos en que se procedió a alterar las estadísticas de precios, así como a remover a los técnicos que desaprobaban tales prácticas, están documentados en la denuncia judicial realizada por el Centro de Estudios Legales y Sociales (CELS, 2009).

${ }^{3}$ El último aspecto mencionado refiere a la correlación entre la posición de un hogar en el ordenamiento según ingresos totales y la posición del mismo en el ordenamiento según un ingreso determinado. Es decir, si los hogares mejor ubicados en la distribución general son aquellos que más reciben ingresos de un determinada fuente, entonces la fuente correspondiente tiene una correlación positiva con la distribución general y, por ende, es de esperar que el mismo aporte de forma relevante a los niveles de desigualdad existentes. 


$$
D_{P S t}=\mathrm{D}_{P E t}+D_{A D t}
$$

$$
\operatorname{con}(\mathrm{t}=1992,1998, \ldots \ldots 2012)
$$

En donde $G$ representa el coeficiente de desigualdad de Gini de ingresos familiares. $D_{\text {ITF(netot) }}$ simboliza la desigualdad generada por los ingresos de los hogares neto de jubilaciones/pensiones y programas sociales de protección contra la pobreza. A su vez, $D_{S S t}$ y $D_{P S t}$ representan - respectivamente - la desigualdad generada por los ingresos obtenidos a través de jubilaciones y pensiones (seguridad social) y mediante la percepción de programas sociales. La desigualdad que se deriva de los programas sociales de asistencia directa y protección al desempleo se conforma, a su vez, por la ocasionada por los programas de empleo $\mathrm{D}_{P E t}$ y la correspondiente a los programas de transferencias no laborales o asistencia directa $D_{A D t}$. El subíndice $t$ simboliza el tiempo.

Por lo tanto, este método no sólo constituye una herramienta útil para estimar el sentido y nivel en que cada una de las fuentes de ingresos contribuye a determinar el coeficiente de Gini y dar forma a su evolución, sino también para explicar de manera dinámica los factores distributivos subyacentes que están detrás de un determinado valor del índice y de sus cambios en el tiempo. Para ello, dado que la ecuación de descomposición asume la forma expresada en (1), según demuestra Cortes (2000), el cambio entre dos índices de Gini que expresen diferencias temporales, uno referido al tiempo $t\left(\mathrm{G}_{\mathrm{t}}\right)$ y otro al tiempo $0\left(\mathrm{G}_{0}\right)$, puede descomponerse de acuerdo con la siguiente ecuación: ${ }^{4}$

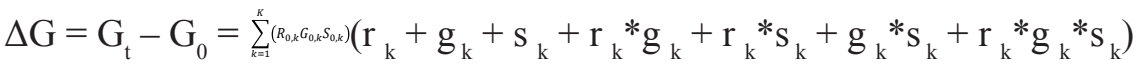

donde $(\mathrm{k}=1,2, \ldots ., \mathrm{K}) \mathrm{y}(\mathrm{t}=1992,1998, \ldots \ldots, 2012)$

Según esta ecuación (3), la contribución de una determinada fuente al cambio general de la desigualdad estará determinada por: (i) los cambios en la correlación entre la distribución del ingreso familiar de un determinado origen con respecto a la distribución total del ingreso (R); (ii) el cambio en la concentración relativa del ingreso por parte de cada fuente considerada (S); (iii) los cambios que registre la desigualdad al interior de

\footnotetext{
${ }^{4}$ Este modelo de descomposición temporal que permite calcular la contribución de los componentes $\mathrm{R}_{k}, \mathrm{~S}_{k}$ y $\mathrm{G}_{k}$ al cambio temporal del coeficiente de Gini fue desarrollado por Cortés (2000) con el objetivo de analizar los cambios ocurridos en la distribución del ingreso en México en épocas de estabilización y reforma económica. Este mismo modelo de descomposición temporal fue aplicado por Salvia (2012) para el caso argentino.
} 
cada fuente (G); y (iv) el efecto de cada una de las correlaciones de primer y segundo orden entre estos factores. En nuestro caso, el factor común que reúne la suma $\left(R_{0, k} G_{0, k} S_{0, k}\right)$ representa la magnitud del aporte de cada una de estos factores a la formación del índice de Gini del ingreso familiar en el tiempo base. Las variables $r_{k,}, g_{k}$ y $s_{k}$ son las tasas de crecimiento lineales simples para cada fuente de la correlación de Gini, de los índices de intradesigualdad y de las participaciones de las fuentes en el ingreso monetario, respectivamente (Anexo 2, Anexo 3, Anexo 4, Anexo 5, Anexo 6, Anexo 7).

Anexo 2: Componentes determinantes del coeficiente de Gini y descomposición del índice según fuentes de ingreso seleccionadas. Gran Buenos Aires, Argentina: 1992, en puntos del Gini

\begin{tabular}{lrrrrr}
\hline & & & & $\begin{array}{r}\text { Contrib. } \\
\text { Abs }\end{array}$ & $\begin{array}{r}\text { Contrib. } \\
\text { relativa }\end{array}$ \\
\hline ITF / Neto de transferencias & 0.9052 & 0.9640 & 0.4820 & 0.4206 & 100.7 \\
Jubilaciones o pensiones & 0.0916 & -0.0669 & 0.7932 & -0.0049 & -1.2 \\
Programas sociales & 0.0033 & 0.5458 & 0.9947 & 0.0018 & 0.4 \\
Programas de empleo & 0.0002 & 0.1931 & 0.9989 & 0.0000 & 0.0 \\
Programas de asistencia directa & 0.0031 & 0.5682 & 0.9954 & 0.0018 & 0.4 \\
Total & & & & 0.4175 & 100.0 \\
\hline Fuente: elaboración propia con base en datos de la EPH "puntual" / INDEC (GBA, Mayo \\
1992).
\end{tabular}

Anexo 3: Componentes determinantes del coeficiente de Gini y descomposición del índice según fuentes de ingreso seleccionadas. Gran Buenos Aires, Argentina: 1998, en puntos del Gini

\begin{tabular}{|c|c|c|c|c|c|}
\hline & $\mathrm{S}$ & $\mathrm{R}$ & $\mathrm{G}$ & $\begin{array}{r}\text { Contrib. } \\
\text { Abs } \\
\end{array}$ & $\begin{array}{r}\text { Contrib. } \\
\text { relativa } \\
\end{array}$ \\
\hline ITF / Neto de transferencias & 0.8789 & 0.9373 & 0.5414 & 0.4460 & 96.3 \\
\hline Jubilaciones o pensiones & 0.1172 & 0.1759 & 0.8335 & 0.0172 & 3.7 \\
\hline Programas sociales & 0.0040 & 0.0202 & 0.9872 & 0.0001 & 0.0 \\
\hline Programas de empleo & 0.0016 & -0.2908 & 0.9903 & -0.0005 & -0.1 \\
\hline Programas de asistencia directa & 0.0023 & 0.2351 & 0.9953 & 0.0005 & 0.1 \\
\hline Total & & & & 0.4633 & 100.0 \\
\hline
\end{tabular}


Anexo 4: Componentes determinantes del coeficiente de Gini y descomposición del índice según fuentes de ingreso seleccionadas. Gran Buenos Aires, Argentina: 2003 (mayo), en puntos del Gini

\begin{tabular}{lrrrrr}
\hline & & & & Contrib. Contrib. \\
& $\mathrm{S}$ & $\mathrm{R}$ & $\mathrm{G}$ & Abs & relativa \\
\hline ITF / Neto de transferencias & 0.8466 & 0.9381 & 0.5816 & 0.4619 & 95.7 \\
Jubilaciones o pensiones & 0.1276 & 0.2524 & 0.8285 & 0.0267 & 5.5 \\
Programas sociales & 0.0258 & -0.2461 & 0.9120 & -0.0058 & -1.2 \\
Programas de empleo & 0.0153 & -0.4847 & 0.9084 & -0.0067 & -1.4 \\
Programas de asistencia directa & 0.0105 & 0.0946 & 0.9667 & 0.0010 & 0.2 \\
Total & & & & 0.4828 & 100.0 \\
\hline Fuente: elaboración propia con base en datos de la EPH "puntual" / INDEC (GBA Mayo \\
2003).
\end{tabular}

Anexo 5: Componentes determinantes del coeficiente de Gini y descomposición del índice según fuentes de ingreso seleccionadas. Gran Buenos Aires, Argentina: 2003 (3er trimestre), en puntos del Gini

\begin{tabular}{lrrrrr}
\hline & & & & $\begin{array}{r}\text { Contrib. } \\
\text { Abs }\end{array}$ & $\begin{array}{r}\text { Contrib. } \\
\text { Relativa }\end{array}$ \\
\hline ITF / Neto de transferencias & 0.8627 & 0.9465 & 0.5737 & 0.4685 & 93.5 \\
Jubilaciones o pensiones & 0.1233 & 0.3468 & 0.8471 & 0.0362 & 7.2 \\
Programas sociales & 0.0140 & -0.2884 & 0.9192 & -0.0037 & -0.7 \\
Programas de empleo & 0.0090 & -0.3811 & 0.9370 & -0.0032 & -0.6 \\
Programas de asistencia directa & 0.0050 & -0.1016 & 0.9749 & -0.0005 & -0.1 \\
Total & & & & 0.5010 & 100.0 \\
\hline
\end{tabular}

Fuente: elaboración propia con base en datos de la EPH "continua" / INDEC (GBA, tercer trimestre de 2003).

Anexo 6: Componentes determinantes del coeficiente de Gini y descomposición del índice según fuentes de ingreso seleccionadas. Gran Buenos Aires, Argentina: 2007, en puntos del Gini

\begin{tabular}{lrrrrr}
\hline & & & & $\begin{array}{r}\text { Contrib. } \\
\text { Abs }\end{array}$ & $\begin{array}{c}\text { Contrib. } \\
\text { Relativa }\end{array}$ \\
\hline ITF / Neto de transferencias & 0.8820 & 0.9447 & 0.5025 & 0.4187 & 99.2 \\
Jubilaciones o pensiones & 0.1115 & 0.0600 & 0.8171 & 0.0055 & 1.3 \\
Programas sociales & 0.0065 & -0.3189 & 0.9440 & -0.0020 & -0.5 \\
Programas de empleo & 0.0011 & -0.3653 & 0.9876 & -0.0004 & -0.1 \\
Programas de asistencia directa & 0.0054 & -0.3031 & 0.9541 & -0.0016 & -0.4 \\
Total & & & & 0.4222 & 100.0 \\
\hline $\begin{array}{l}\text { Fuente: elaboración propia con base en datos de la EPH “continua" / INDEC (GBA, segundo } \\
\text { trimestre de 2007). }\end{array}$
\end{tabular}


Anexo 7: Componentes determinantes del coeficiente de Gini y descomposición del índice según fuentes de ingreso seleccionadas. Gran Buenos Aires, Argentina: 2012, en puntos del Gini

\begin{tabular}{lrrrrr}
\hline & & & & Contrib. & Contrib. \\
& $\mathrm{S}$ & $\mathrm{R}$ & $\mathrm{G}$ & Abs & Relativa \\
\hline ITF / Neto de transferencias & 0.8428 & 0.9217 & 0.4950 & 0.3845 & 97.7 \\
Jubilaciones o pensiones & 0.1473 & 0.0910 & 0.7767 & 0.0104 & 2.6 \\
Programas sociales & 0.0099 & -0.1334 & 0.9342 & -0.0012 & -0.3 \\
Programas de empleo & 0.0006 & 0.0236 & 0.9976 & 0.0000 & 0.0 \\
Programas de asistencia directa & 0.0092 & -0.1444 & 0.9337 & -0.0012 & -0.3 \\
Total & & & & 0.3937 & 100.0 \\
\hline
\end{tabular}

Fuente: elaboración propia con base en datos de la EPH "continua" / INDEC (GBA, segundo trimestre de 2012). 